\title{
RECONSTRUCTING PLANE QUARTICS FROM THEIR INVARIANTS
}

\author{
REYNALD LERCIER, CHRISTOPHE RITZENTHALER, AND JEROEN SIJSLING
}

\begin{abstract}
We present an explicit method that, given a generic tuple of Dixmier-Ohno invariants, reconstructs a corresponding plane quartic curve.
\end{abstract}

\section{INTRODUCTION}

Invariant theory played a central role in nineteenth-century algebra and geometry, and the natural action of linear groups on spaces of homogenous polynomials in several variables, or forms, was one of its principal areas of focus. Nowadays, motivated by computational applications to cryptography, robotics, coding theory, and experimental mathematics, this theory has come to a renaissance. It is a source of many questions with an explicit or computational orientation. One of these questions, the reconstruction of ternary quartics (i.e., forms of degree 4 in 3 variables) from their invariants, is the central theme of our paper.

Of old, the group $\mathrm{SL}_{2}(\mathbb{C})$ and its action on the ring $R_{2, n}$ of binary forms $b\left(z_{1}, z_{2}\right)$ of degree $n \geq 2$ over $\mathbb{C}$ have received the lion's share of attention. One reason is the remarkable formalism developed by Gordan in 1868 [16] and by Hilbert in 1897 [18] to compute a finite set of generators of the ring of invariants $\mathbb{C}\left[R_{2, n}\right]^{\mathrm{SL}_{2}(\mathbb{C})}$ of $R_{2, n}$. The implementations of their approaches have so far led to the determination of a set of generators (called fundamental invariants) of these invariant rings for $n \leq 10$; here we refer to $[5,6,16,41,42]$.

This algebraic problem has a geometric counterpart. Given a binary form $b$ of even degree $n$ with simple roots, we can consider the degree 2 cover $\mathbf{X}$ of $\mathbb{P}_{\mathbb{C}}^{1}$ defined by the equation $\mathbf{X}: y^{2}=b\left(z_{1}, z_{2}\right)$ in the weighted projective space with coordinates $z_{1}, z_{2}, y$ and weights $1,1, n$. Since isomorphisms between hyperelliptic curves are induced by the action of $\mathrm{GL}_{2}(\mathbb{C})$, the values of fundamental invariants of $R_{2, n}$ on the form $b$ define a point in a certain weighted projective space which characterizes the geometric isomorphism class of $\mathrm{X}$. For $n=4$ an affine coordinate on this space is given by the classical Weierstrass $j$-invariant; for larger even $n$ this construction gives rise to an explicit embedding of the moduli space of hyperelliptic curves of genus $n / 2-1$ into a weighted projective space.

When we broaden our scope to consider the action of $\mathrm{SL}_{3}(\mathbb{C})$ on the ring $R_{3, n}$ of ternary homogenous polynomials $F$ of degree $n$, explicit sets of generators are known only for $n \leq 4$. While the cases $n=2,3$ were known classically, the case

Date: June 20, 2016.

2010 Mathematics Subject Classification. 13A50, 14L24, 14H10, $14 \mathrm{H} 25$.

Key words and phrases. plane quartic curves; invariant theory; Dixmier-Ohno invariants; moduli spaces; reconstruction.

The first two authors acknowledge support from the CysMoLog "défi scientifique émergent" of the Université de Rennes 1. 
$n=4$ was completely settled only recently, by Dixmier [9] and Ohno [28] (but see also $[11,15])$. The work of these authors shows that the ring $\mathbb{C}\left[R_{3,4}\right]^{\mathrm{SL}_{3}(\mathbb{C})}$ is generated by 13 elements, usually called the Dixmier-Ohno invariants of ternary quartics. We discuss these results in Section 1.2.

The cases $n=2,3$ correspond to curves of genus 0 and 1, which can at least equally conveniently be seen as degree 2 covers of the projective line. Upon passing to the case $n=4$, this changes drastically. The associated plane quartic curve $\mathrm{X}: F\left(x_{1}, x_{2}, x_{3}\right)=0$ is now a non-hyperelliptic curve of genus 3 . Two plane quartics that are smooth (as is generically the case) are isomorphic if and only if the corresponding ternary quartic forms are equivalent under the action of $\mathrm{GL}_{3}(\mathbb{C})$.

In this algebraic-geometric setup

$$
\begin{aligned}
\{\text { binary } / \text { ternary forms }\}_{/ G L} & \longleftrightarrow \text { classical invariants }\} \\
\{\text { curves of genus } g\}_{/ \simeq} & \longleftrightarrow \text { space of coordinates }\}
\end{aligned}
$$

we have so far only indicated how to go from left to right. However, there are many areas where one is also interested in the reconstruction of a curve from given invariants. Here one can think of the construction of CM curves [8] but also of finding curves with many points [33] or of experiments in arithmetic statistics [23]. For binary forms of degrees $n \in\{4,6,8\}$, that is to say, for elliptic curves and for hyperelliptic curves of genus $\leq 3$, this reconstruction is indeed possible over any algebraically closed field of characteristic $p$ for $n=4$ and 6 and for fields of characteristic 0 or $p>7$ if $n=8$. The cases $2 \leq p \leq 7$ were analyzed in [2]; reconstruction turns out to be possible for $p \in\{3,7\}$, and for $p=2$ an orbitseparating set of invariants can be constructed.

The main tool in this context is a method due to Mestre [27] (see the introduction of [22] for more details). It is based on formulas that go back to Clebsch [7, $\S 103$ ] and uses a generalization of invariants called covariants (see Definition 1.3). Roughly speaking, starting from three covariants of order 2 , one constructs a conic $\boldsymbol{Q}$ and a plane curve $\boldsymbol{H}$ of degree $n / 2$. The coefficients of the curves $\boldsymbol{Q}$ and $\boldsymbol{H}$ are invariants, and can in particular be expressed in terms of the fundamental invariants mentioned above. This means that when given values of the fundamental invariants, we can construct the corresponding specializations $Q$ and $H$ of $\boldsymbol{Q}$ and $\boldsymbol{H}$. If the resulting specialization $Q$ is non-singular, then we can build the degree 2 cover of $Q$ that ramifies at the $n$ points where $Q$ and $H$ intersect. This cover will then have the requested fundamental invariants. Note that the singularity of the conic $\boldsymbol{Q}$ is in turn described by the vanishing of a generically non-zero invariant. Therefore this method furnishes us with a way to reconstruct generic hyperelliptic curves of genus at most 3 from their invariants. When using enough distinct covariants, this reconstruction in fact becomes possible for any hyperelliptic curve of genus at most $3, c f .[22]$.

For ternary forms, the reconstruction problem is more complicated. Of course in low degree the obstacles are not very extensive yet. Certainly the case of ternary quadrics is manageable enough, as all of these are geometrically isomorphic to $\mathbb{P}^{1}$. The case of ternary cubics can be dealt with by considering these genus 1 curves as an elliptic curve and reconstructing this curve from its Weierstrass $j$-invariant (see [38, 4.5, p.173] and [10, Sec.10.3]). The case of ternary quartics, however, has been open for a long time. This is in part because the formulas by Clebsch 
that are used in Mestre's method remain mysterious, in the sense that no general encompassing formalism for their derivation has been found yet. Another complicating factor in the ternary quartic case is the high dimension of the underlying geometry of the space of ternary quartics. Of course, Katsylo [20] showed that the moduli space of plane quartics is rational. As such, by the triviality of the descent obstruction for generic plane quartics there should exist a quartic curve over the purely transcendental field $\mathbb{C}\left(\mathbb{P} \operatorname{Sym}^{4}\left(V^{*}\right)\right)^{\mathrm{SL}_{3}(\mathbb{C})}$ whose coefficients are functions in the Dixmier-Ohno invariants. However, the generators of this field are not known explicitly, and if the binary case [26] is a fair indication, the corresponding invariants are likely to be rather unmanageable, which leaves even less hope for writing down a such a generic quartic curve.

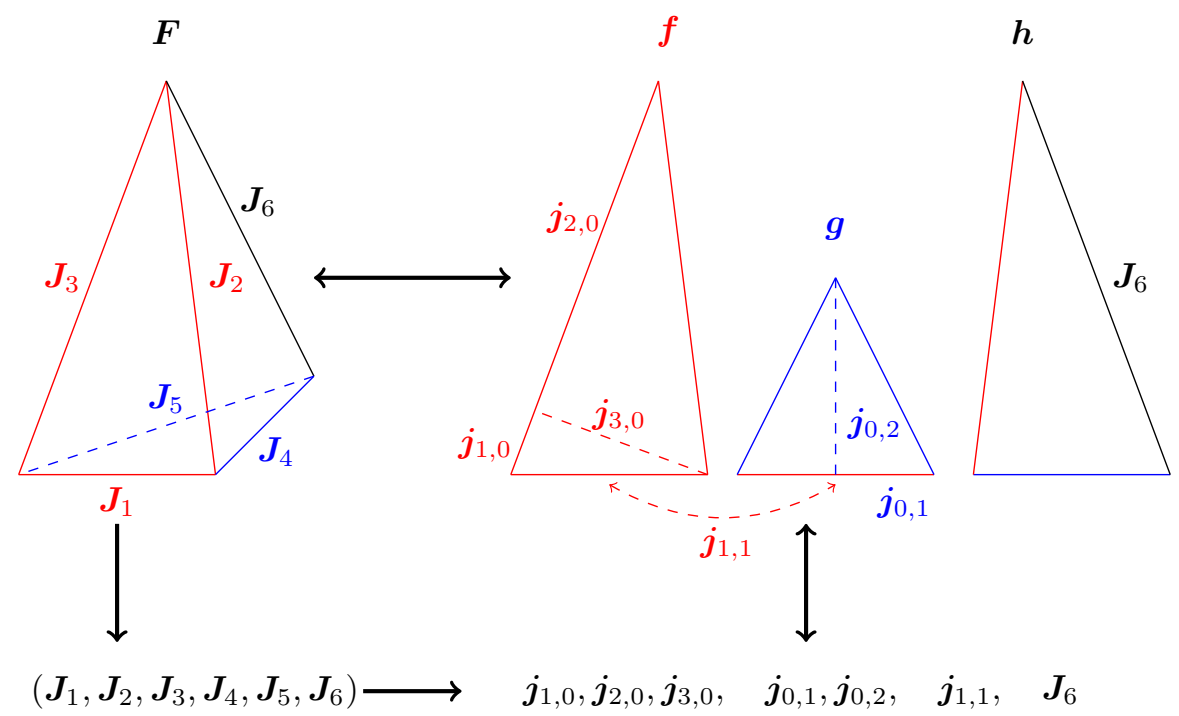

FiguRE 1. Geometric analogue of the reconstruction strategy

Our strategy therefore takes a detour and involves several steps. We illustrate them in Figure 1. This picture is to be interpreted as follows. On the left hand side, we consider a "universal" tetrahedron $\boldsymbol{F}$; this universality is reflected in our notation with a boldface, as for the "universal" curves $\boldsymbol{Q}$ and $\boldsymbol{H}$ considered in Mestre's method above. This tetrahedron $\boldsymbol{F}$ is the analogue of the ternary quartic forms in which we are interested. The lengths of the sides of $\boldsymbol{F}$ correspond to the invariants of this form. Note the analogy: the side lengths of a tetrahedron are indeed invariant under isometry. We let $\boldsymbol{J}_{i}$ be a set of side lengths from which our tetrahedron $\boldsymbol{F}$ can be reconstructed; these are the analogue of the Dixmier-Ohno invariants.

We reconstruct $\boldsymbol{F}$ from the $\boldsymbol{J}_{i}$ by an indirect route by taking a counterclockwise detour through a composition of three other arrows, which are defined in the following way. The top arrow is the extraction of three of the faces $\boldsymbol{f}, \boldsymbol{g}, \boldsymbol{h}$ of $\boldsymbol{F}$ and conversely, we assume that we have an explicit way to reconstruct $\boldsymbol{F}$ from these faces. Moreover, these faces are in turn characterized by certain lengths $\boldsymbol{j}_{0, b}$ and $\boldsymbol{j}_{a, 0}$, and these lengths additionally satisfy some mutual relations. The face 
length $\boldsymbol{j}_{1,1}$ is the analogue of what we will later call a joint invariant. It is easier to reconstruct the faces $\boldsymbol{f}, \boldsymbol{g}, \boldsymbol{h}$ in a 2-dimensional space (a process indicated by the rightmost arrow), so that we see that we can indeed hope to reconstruct $\boldsymbol{F}$ as long as we know how to obtain the joint invariants $\boldsymbol{j}_{a, b}$ from the invariants $\boldsymbol{J}_{i}$. This link between the invariants is represented by the bottom arrow.

In the context of ternary quartic forms, the "faces" $\boldsymbol{f}, \boldsymbol{g}$ and $\boldsymbol{h}$ mentioned above are the analogues of three binary forms $\boldsymbol{b}_{8}, \boldsymbol{b}_{4}$ and $\boldsymbol{b}_{0}$ of respective degree 8,4 and 0 (so that $\boldsymbol{b}_{0}$ is a constant polynomial in the invariants). The group $\mathrm{SL}_{2}(\mathbb{C})$ has a simultaneous action on these forms. Olive [29] has recently determined a corresponding set of fundamental invariants $\boldsymbol{j}_{d_{1}, d_{2}}$. Among these joint invariants are a set of fundamental invariants $\boldsymbol{j}_{d_{1}, 0}$ of the octic form $\boldsymbol{b}_{8}$, similar fundamental invariants $\boldsymbol{j}_{0, d_{2}}$ of the quartic $\boldsymbol{b}_{4}$, and the form $\boldsymbol{b}_{0}$ itself, but the invariant algebra contains many more "cross-terms" beyond these.

The correspondence between $\boldsymbol{F}$ and the triple $\underline{\boldsymbol{b}}=\left(\boldsymbol{b}_{8}, \boldsymbol{b}_{4}, \boldsymbol{b}_{0}\right)$ is induced by a classical isomorphism between $\mathrm{SL}_{2}(\mathbb{C}) /\{ \pm 1\}$ and the special orthogonal group $\mathrm{SO}(q)$ of a fixed (co- or contravariant) ternary quadratic form $q$. Using Lie theory, one constructs an isomorphism between $\mathbb{C}\left[R_{3,4}\right]^{\mathrm{SO}(q)}$ and the ring of invariants $\mathbb{C}\left[R_{2,8} \oplus R_{2,4} \oplus R_{2,0}\right]^{\mathrm{SL}_{2}(\mathbb{C})}$ for the diagonal action of $\mathrm{SL}_{2}(\mathbb{C})$. This idea was exploited by Katsylo [20] and used in a more explicit way by Van Rijnswou [40]. We refer to Section 2.1 for details on this part of our argument.

We are interested in invariants under the whole group $\mathrm{SL}_{3}(\mathbb{C})$, not merely those of $\mathrm{SO}(q)$. Fortunately, it is possible to reduce the study of the former invariants to that of the latter by using the notion of $(G, H)$-sections (see Definition 2.3). Roughly speaking, one can show that a generic quartic $F$ is $\mathrm{SL}_{3}(\mathbb{C})$-equivalent to one in the set $Z$ of quartics whose quadratic contravariant $\boldsymbol{\rho}(F)$ (as defined in (1.23)) is a non-zero multiple $u \cdot q$ of the chosen standard quadratic form $q$. Moreover, up to scalar multiplication two generic quartics in $\mathrm{Z}$ are equivalent under the action of $\mathrm{SL}_{3}(\mathbb{C})$ if and only if they are equivalent under the action of $\mathrm{SO}_{3}(q)$.

As was known to Katsylo (but see also the first part of Proposition 2.5) this means that the function field $\mathbb{C}\left(R_{3,4}\right)^{\mathrm{SL}_{3}(\mathbb{C})}$ is isomorphic to $\mathbb{C}(\mathrm{Z})^{\mathrm{SO}_{3}(q)}$. We can then use the correspondence between $F$ and the triple $\underline{b}=\left(b_{8}, b_{4}, b_{0}\right)$. After this, we show that one can actually control the denominator of this expression, as well as the degree of the numerator. This is achieved by combining a regularity statement (see the second part of Proposition 2.5) with a detailed study of a transformation matrix $T$ that transforms a generic quadric to one whose covariant is of the form $u \cdot q$ (see Section 2.3) and a fundamental relation between $\boldsymbol{b}_{0}$, the Dixmier-Ohno invariants $\boldsymbol{I}_{9}, \boldsymbol{I}_{12}$ and the determinant of $T$ (see Lemma 2.12).

Our final theoretic description of the numerator and denominator is given in Theorem 2.16, for which we present two different proofs. Using interpolation then allows us to recover explicit expressions for the joint invariants in terms of the Dixmier-Ohno invariants, as is described further in Section 2.8.

The final step is to actually perform the reconstruction of a triple $\left(b_{8}, b_{4}, b_{0}\right)$ once values of the joint invariants are given. We first show that if $F$ is a stable quartic (which is in particular the case for quartics with non-zero discriminant) and $\boldsymbol{I}_{12}(F) \neq 0$, then the corresponding triple $\left(b_{8}, b_{4}, b_{0}\right)$ is in the stable locus of the action of $\mathrm{SL}_{2}(\mathbb{C})$ and therefore its orbit is uniquely determined by its joint invariants (see Theorem 3.1). Reconstruction is therefore theoretically possible 
under these hypotheses; it remains to show how to do this explicitly in the generic situation.

Our reconstruction algorithm in Section 3.2 starts from given Dixmier-Ohno invariants defined over field $k$ of characteristic 0 . In order to avoid working over a cubic extension of the field of definition of the invariants, we normalize the invariant $b_{0}$ so it becomes equal to the Dixmier-Ohno invariant $I_{9}$ and then we use the explicit relations of Section 2.8 to get the corresponding joint invariants $j_{d_{1}, d_{2}}$. We then use the methods from [22] for reconstructing a stable binary octic $b_{8}$ from given Shioda invariants, which can be derived from the $j_{i, 0}$ (see Remark 1.8). Note that this step, which uses the Mestre's method, will in general require a quadratic extension of the base field $k$. One can then use the values $j_{d, 1}$ of the joint invariants $\boldsymbol{j}_{d, 1}$ which are linear in the coefficients of $b_{4}$, to determine the coefficients of $b_{4}$ by solving a linear system. Finally, one transforms back the triple $\left(b_{8}, b_{4}, b_{0}\right)$ by a linear isomorphism $\ell^{*}$ (given in (2.17)) to find a ternary quartic $F$ which Dixmier-Ohno invariants are projectively equal to the given ones as a point in a weighted projective space. We briefly indicate in Section 3.3 how one can perform a Galois descent if one wishes to find an $F$ over $k$, which in fact is generically possible. Finally, a form $F$ over $k$ whose Dixmier-Ohno invariants are projectively equivalent to the given ones can generically also be scaled over $k$ to obtain an exact equality of invariants.

The implementation of our results that was used when writing this article can be found at [24].

A number of open problems remain. A first of these is to remove our genericity assumptions; one would certainly like to be able to deal with the quartics in the locus $\boldsymbol{I}_{12}=0$ as well, and more generally, to be able to perform the reconstruction for any tuple of Dixmier-Ohno invariants corresponding to a stable quartic. Another open problem is the extension of our theory to fields of positive characteristic, for which it is not even clear when the Dixmier-Ohno invariants are still a fundamental set.

Acknowledgments. We would like to thank the participants of the working group TEDI, and in particular Boris Kolev and Marc Olive, for their interest and for the many useful discussions.

Notation and conventions. While we recall most conventions at the beginning of the relevant sections, here are some general conventions to which we adhere in this article.

In what follows, $K$ is an algebraically closed base field, supposed to be of characteristic 0. Forms over $K$ are denoted with Roman letters, which are capitalized when dealing with ternary forms. The universal forms of forms, as well as those of their covariants, are denoted in boldface Roman letters, so that we for example see individual ternary forms $F$ as incarnations of the universal ternary quartic form $\boldsymbol{F}$. Conversely, when specializing at a given form over $K$, this boldface is removed, so that the value of a covariant $\boldsymbol{C}$ at a form $F$ over $K$ is denoted by $C$. Contravariants and their values are denoted by Greek letters, so that a contravariant $\gamma$ has a specialization $\gamma=\gamma(F)$ at a form $F$ over $K$.

For the sake of clarity, we denote group actions with an intermediate dot, so that we write $g . s$ where one would often merely find $g s$. 


\section{INVARIANT THEORY}

1.1. Formalism. In this section we describe the actions of linear groups that we will need in the rest of the article, as well as the formalism needed to deal with invariants, covariants, and contravariants. This exposition is adapted to our needs and therefore not in any sense complete; other general discussions of the theory of invariants and covariants can be found in [21, §1] and [10, Lecture 5].

Given a finite-dimensional vector space $V$ over an algebraically closed field $K$, let $V^{*}=\operatorname{Hom}(V, K)$ be its dual. Since $K$ is infinite, homogeneous polynomial functions on $V$ can be naturally identify with forms, which are defined as follows.

Definition 1.1. A form over $K$ is an element $F$ of a vector space $\operatorname{Sym}^{n}\left(V^{*}\right)$, where $V$ is a finite-dimensional vector space over $K$. Given a form $F$, its arity (or more colloquially its number of variables) is the dimension $m$ of $V$. The degree of $F$ is denoted by $n$.

Given $V$, we also define the polynomial ring over $V$ to be the graded ring

$$
K[V]=\bigoplus_{n \geq 0} \operatorname{Sym}^{n}\left(V^{*}\right),
$$

and we define the affine space $\mathbb{A}_{V}$ over $V$ to be the variety $\operatorname{Spec} K[V]$. In particular, the functor that to a vector space associates the corresponding affine space is covariant.

In this article, we will consider group actions on several vector spaces and duals. We will occasionally want to switch actions from left to right, and as such, given a left (resp. right) action of a group $G$ on a vector space $V$, we convert it into a right (resp. left) action by setting

$$
v \cdot T:=T^{-1} . v \quad\left(\operatorname{resp} . T \cdot v:=v \cdot T^{-1}\right)
$$

for $T \in G$ and $v \in V$.

A subgroup $G$ of GL $(V)$ has a natural left action on $V$. This induces a natural right action on $V^{*}$; for $x \in V^{*}$ and $T \in G$ we have $x . T=x \circ T$. These actions induce further natural actions on $\operatorname{Sym}^{n}(V)$ and $\operatorname{Sym}^{n}\left(V^{*}\right)$.

Choosing a basis $\underline{v}=\left(v_{1}, \ldots, v_{m}\right)$ of $V$, we get a dual basis $\underline{x}=\left(x_{1}, \ldots, x_{m}\right)=$ $\left(v_{1}^{*}, \ldots, v_{m}^{*}\right)$ of $V^{*}$. Given $n$ and a list $I=\left(i_{1}, \ldots, i_{m}\right)$ of $m$ non-negative integers the sum of which is $n$, we denote $x^{I}=x_{1}^{i_{1}} \cdots x_{m}^{i_{m}} \in \operatorname{Sym}^{n}\left(V^{*}\right)$. The elements $x^{I}$ form a basis of $\operatorname{Sym}^{n}\left(V^{*}\right)$, and we denote the elements of the corresponding dual basis $\underline{a}$ by $a_{I} \in \operatorname{Sym}^{n}(V)$. For a form $F$, we have $\left(x^{I}\right)^{*}(F)=a_{I}(F)$, so that we can write $F=\sum a_{I}(F) x^{I}$ and identify the values $a_{I}(F) \in K$ with the coefficients of $F$ in this expression.

A choice of basis for $V$ also allows us to identify an element $T$ of $\operatorname{GL}(V)$ with a matrix $[T]=\left(t_{i, j}\right)_{i, j=1}^{m}$. With this notation, the result T. $v_{i}$ of having $T$ act on the left on the $i$ th vector $v_{i}$ of the basis $\underline{v}$ of $V$ corresponds to the $i$ th column of the matrix $[T]$. By duality, the result $x_{i} . T$ of having $T$ act on the right on the $i$ th vector $x_{i}$ of the dual basis $\underline{x}$ of $V^{*}$ corresponds to the $i$ th row of the matrix $[T]$, or alternatively to the $i$ th column of the transpose of $[T]$. In a formula, we have

$$
\begin{gathered}
\text { T. } v_{i}=t_{1, i} v_{1}+\cdots+t_{m, i} v_{m} \\
\left(\operatorname{resp.} x_{i} \cdot T=t_{i, 1} x_{1}+\cdots+t_{i, m} x_{m}\right) .
\end{gathered}
$$


We will abbreviate

$$
T \cdot \underline{v}=\left(T \cdot v_{1}, \ldots, T \cdot v_{m}\right) \quad\left(\operatorname{resp} \cdot \underline{x} \cdot T=\left(x_{1} \cdot T, \ldots, x_{m} \cdot T\right)\right) .
$$

Remark 1.2. The substitution $\underline{x} . T$ corresponding to the right action of $T$ on $\underline{x}$ (and that also induces the right action of $T$ on the symmetric powers $\operatorname{Sym}^{n}\left(V^{*}\right)$ ) is in fact encoded in the formal matrix product

$$
\left(\begin{array}{ccc}
t_{1,1} & \ldots & t_{1, n} \\
\vdots & \ddots & \vdots \\
t_{n, 1} & \ldots & t_{n, n}
\end{array}\right) \times\left(\begin{array}{c}
x_{1} \\
\vdots \\
x_{n}
\end{array}\right) .
$$

Note how in (1.5) the right action on the dual is obtained by using variables instead of values for the usual left action of $[T]$ on column vectors; this is a general principle when dualizing. The substitution T. $\underline{v}$ corresponding to the left action of $T$ on $V$ can be obtained by multiplying the formal row vector corresponding to $\underline{v}$ on the right by $T$ instead.

The individual forms $F \in \operatorname{Sym}^{n}\left(V^{*}\right)$ can be seen as the incarnations of a universal form, which we will denote by $\boldsymbol{F}$. In coordinates, this is the form

$$
\boldsymbol{F}:=\boldsymbol{F}(\underline{a}, \underline{x}):=\boldsymbol{F}\left(\left(a_{I}\right),\left(x^{I}\right)\right)=\sum_{I: \sum I=n} a_{I} x^{I} \in \operatorname{Sym}^{n}(V) \otimes \operatorname{Sym}^{n}\left(V^{*}\right) .
$$

The duality $\left\langle x^{I}, T \cdot a_{J}\right\rangle=\left\langle T^{*}\left(x^{I}\right), a_{J}\right\rangle=\left\langle x^{I} . T, a_{J}\right\rangle$ implies the fundamental compatibility

$$
\boldsymbol{F}(T \cdot \underline{a}, \underline{x}):=\boldsymbol{F}\left(\left(T \cdot a_{I}\right),\left(x^{I}\right)\right)=\boldsymbol{F}\left(\left(a_{I}\right),\left(x^{I} \cdot T\right)\right)=: \boldsymbol{F}(\underline{a}, \underline{x} \cdot T) .
$$

This also follows because $\boldsymbol{F}$ corresponds to the canonical bilinear contraction (or evaluation) $\operatorname{Sym}^{n}\left(V^{*}\right) \times \operatorname{Sym}^{n}(V) \rightarrow K$. A form $F$ over $K$ can be obtained from the universal form $\boldsymbol{F}$ by contracting it with the morphism $\operatorname{Sym}^{n}(V) \rightarrow K$ that corresponds to $F \in \operatorname{Sym}^{n}\left(V^{*}\right)$.

Using the coordinate vectors $\underline{a}$ and $\underline{x}$ (resp. $\underline{v}$ ) in what follows will allow us to concretely represent covariant (resp. contravariant) tensors as bihomogeneous polynomials of degree $d$ in the $a_{I}$ and degree $r$ in $x^{I}$ (resp. $v^{I}$ ).

The following notion is a fundamental tool in the study of the representations $\operatorname{Sym}^{n}\left(V^{*}\right)$ of GL( $\left.V\right)$ and its subgroups.

Definition 1.3. A covariant (resp. contravariant) of $\operatorname{Sym}^{n}\left(V^{*}\right)$ is an $\operatorname{SL}(V)$-equivariant homogeneous polynomial map

$$
\begin{array}{r}
C: \operatorname{Sym}^{n}\left(V^{*}\right) \rightarrow \operatorname{Sym}^{r}\left(V^{*}\right) \\
\left(\text { resp. } \gamma: \operatorname{Sym}^{n}\left(V^{*}\right) \rightarrow \operatorname{Sym}^{r}(V)\right) .
\end{array}
$$

The order of $\boldsymbol{C}$ (resp. $\boldsymbol{\gamma}$ ) is defined to be $r$, whereas the degree of $\boldsymbol{C}$ (resp. $\boldsymbol{\gamma}$ ) is its degree (in $\underline{a}$ ) as a homogeneous polynomial map.

An invariant is a covariant (or, for that matter, a contravariant) of order 0 .

Remark 1.4. In a sense, we could have decided only to work with covariants, since after agreeing that $\operatorname{Sym}^{r}\left(V^{*}\right)=\operatorname{Sym}^{-r}(V)$ for $r$ negative, a contravariant becomes nothing but a covariant of negative order. This description would lead to a fully unified description of co- and contravariants and a more concise formalism. We have however rather chosen to follow the classical description, in which co- and contravariants are distinguished. 
At any rate, we have already restricted ourselves to a special case. In general (see $[21, \S 1.4]$ and especially $[10, \S 5.2]$ ), given a representation $V$ of a group $G$, a $W$-covariant of $V$ is a $G$-equivariant homogeneous polynomial map $V \rightarrow W$, where $W$ is another representation of $G$. As was classically the custom, we have restricted ourselves to covariants of the same arity as the original form. Note that in order to study joint covariants (which is in fact needed to determine the joint invariants mentioned at the end of this section) we would have to use the more general notion just mentioned.

We proceed to unwind our definitions. Consider a co- or contravariant as in (1.8), of order $r$ and degree $d$.

(i) We can consider the homogeneous polynomial map (1.8) as an equivariant linear map $\operatorname{Sym}^{d}\left(\operatorname{Sym}^{n}\left(V^{*}\right)\right) \rightarrow \operatorname{Sym}^{r}\left(V^{*}\right)\left(\operatorname{resp} \cdot \operatorname{Sym}^{d}\left(\operatorname{Sym}^{n}\left(V^{*}\right)\right) \rightarrow\right.$ $\operatorname{Sym}^{r}(V)$ ), which in turn is nothing but an invariant tensor

$$
\begin{array}{r}
\boldsymbol{C} \in\left(\operatorname{Sym}^{d}\left(\operatorname{Sym}^{n}(V)\right) \otimes \operatorname{Sym}^{r}\left(V^{*}\right)\right)^{\operatorname{SL}(V)} \\
\text { (resp. } \left.\boldsymbol{\gamma} \in\left(\operatorname{Sym}^{d}\left(\operatorname{Sym}^{n}(V)\right) \otimes \operatorname{Sym}^{r}(V)\right)^{\mathrm{SL}(V)}\right) .
\end{array}
$$

(ii) In this form, the demand that $C$ (resp. $\gamma$ ) be invariant translates into the analogue of (1.7) (but only for transformations by $\mathrm{SL}(V)$; see (1.13) and (1.14) below for the transformation behavior under GL $(V)$ ). Indeed, for $T \in \mathrm{SL}(V)$ the invariance of $\boldsymbol{C}=\boldsymbol{C}(\underline{a}, \underline{x})$ under the diagonal action under $T$ translates to

$$
\begin{array}{r}
C(\underline{a}, \underline{x}) \cdot T=C(\underline{a} \cdot T, \underline{x} \cdot T)=\boldsymbol{C}\left(T^{-1} \cdot \underline{a}, \underline{x} \cdot T\right) \\
\left(\operatorname{resp} . \gamma(\underline{a}, \underline{x}) \cdot T=\gamma(\underline{a} \cdot T, \underline{x} \cdot T)=\gamma\left(T^{-1} \cdot \underline{a}, \underline{x} \cdot T\right)\right) .
\end{array}
$$

Applying $T$ on the factor $\operatorname{Sym}^{n}(V)$ then leads to

$$
\begin{array}{r}
C(T \cdot \underline{a}, \underline{x})=\boldsymbol{C}(\underline{a}, \underline{x} \cdot T) \\
(\operatorname{resp} . \gamma(T \cdot \underline{a}, \underline{v})=\gamma(\underline{a}, \underline{v} \cdot T)) .
\end{array}
$$

(iii) Putting all degrees and orders together, we can also consider $\boldsymbol{C}$ (resp. $\gamma$ ) as a homogeneous element of bidegree $(d, r)$ of the algebra

$$
\begin{gathered}
\left(K\left[\operatorname{Sym}^{n}\left(V^{*}\right)\right] \otimes K[V]\right)^{\mathrm{SL}(V)}=K\left[\operatorname{Sym}^{n}\left(V^{*}\right) \oplus V\right]^{\mathrm{SL}(V)} \\
\left(\text { resp. }\left(K\left[\operatorname{Sym}^{n}\left(V^{*}\right)\right] \otimes K\left[V^{*}\right]\right)^{\mathrm{SL}(V)}=K\left[\operatorname{Sym}^{n}\left(V^{*}\right) \oplus V^{*}\right]^{\mathrm{SL}(V)}\right) .
\end{gathered}
$$

(iv) Or yet differently, by dualizing (i) we see that $C$ (resp. $\gamma$ ) is nothing but an inclusion of the irreducible representation $\operatorname{Sym}^{r}(V)\left(\operatorname{resp} . \operatorname{Sym}^{r}\left(V^{*}\right)\right)$ of $\operatorname{SL}(V)$ into either the finite-dimensional representation $\operatorname{Sym}^{d}\left(\operatorname{Sym}^{n}(V)\right)$ or the infinite-dimensional representation $K\left[\operatorname{Sym}^{n}\left(V^{*}\right)\right]$.

By studying the action of scalar (or diagonal) matrices, we see that the action of elements of $\mathrm{GL}(V)$ is also intertwined, but only up to a scalar. More precisely, for covariants we have

$$
\boldsymbol{C}(F . T)=\operatorname{det}(T)^{(n d-r) / m} \boldsymbol{C}(F) \cdot T,
$$

while for contravariants we have

$$
\gamma(F \cdot T)=\operatorname{det}(T)^{(n d+r) / m} \gamma(F) \cdot T .
$$

The relevant (integral!) quotient $(n d-r) / m(\operatorname{resp} .(n d+r) / m)$ is called the weight of the covariant (resp. contravariant). 
Remark 1.5. The point of view (iv) above that considers covariants and contravariants as subrepresentations is quite valuable when we want to make the theory explicit. Indeed, one can decompose tensor powers $\operatorname{Sym}^{d}\left(\operatorname{Sym}^{n}(V)\right)$ in computer algebra packages such as MAGMA [4] or, much faster, in LIE [39]. Using these packages for ternary quartics (so $\operatorname{dim}(V)=3$ and $n=4$ ) shows that the first nontrivial contravariant of order 2 occurs for $d=4$, in which case there is a unique such covariant up to scaling. This is the contravariant $\boldsymbol{\rho}$ constructed in Section 1.2, which has also been mentioned in passing in the introduction.

The first covariants of order 2 show up in degree $d=5$. In this case the factor $\operatorname{Sym}^{2}(V)$ occurs with multiplicity 2 , so any covariant of degree 5 can uniquely be written as a combination

$$
\lambda_{1} C_{1}+\lambda_{2} C_{2}: \operatorname{Sym}^{4}\left(V^{*}\right) \rightarrow \operatorname{Sym}^{2}\left(V^{*}\right)
$$

for two fixed independent such covariants $\boldsymbol{C}_{1}$ and $\boldsymbol{C}_{2}$ that can be taken to be the covariants $\boldsymbol{T}$ and $\boldsymbol{X}$ from (1.24).

In Section 1.3, we will also consider joint invariants. These arise when considering the action of a subgroup $G$ of $\mathrm{GL}(V)$ on a reducible representation, in our case direct sums of vector spaces

$$
S=\operatorname{Sym}^{n_{1}}\left(V^{*}\right) \oplus \ldots \oplus \operatorname{Sym}^{n_{t}}\left(V^{*}\right) .
$$

We have $G$ act diagonally on $S$, so that

$$
\left(F_{1}, \ldots, F_{t}\right) \cdot T=\left(F_{1} \cdot T, \ldots, F_{t} \cdot T\right)
$$

for $T \in G$ and $\left(F_{1}, \ldots, F_{t}\right) \in S$. This gives rise to the invariant algebra $K[S]^{G}$, which has a natural grading by multi-degree in the coefficients of the forms $F_{i}$. A form that is pure of multi-degree $\left(d_{1}, \ldots, d_{t}\right)$ is denoted by $\boldsymbol{j}_{d_{1}, \ldots, d_{t}}$. We will call $d=d_{1}+\ldots+d_{t}$ the total degree of such an invariant.

1.2. Dixmier-Ohno invariants. In this section we briefly recall the construction and notation of some of the invariants, covariants and contravariants of ternary quartics. We refer to $[9,11,15]$ for more details.

Let $V$ be a vector space of dimension 3 over $K$ with basis $v_{1}, v_{2}, v_{3}$ and corresponding dual basis $x_{1}, x_{2}, x_{3}$. Let

$$
D: K\left[x_{1}, x_{2}, x_{3}\right] \times K\left[v_{1}, v_{2}, v_{3}\right] \rightarrow K\left[x_{1}, x_{2}, x_{3}\right]
$$

be the differential operator that extends the linear contraction pairing $\left(v_{i}, x_{j}\right) \rightarrow \delta_{i j}$ by associating to a monomial $v_{1}^{i_{1}} v_{2}^{i_{2}} v_{3}^{i_{3}}$ of degree $m$ the operator

$$
\frac{\partial^{m}}{\partial x_{1}^{i_{1}} \partial x_{2}^{i_{2}} \partial x_{3}^{i_{3}}} .
$$

If $Q\left(x_{1}, x_{2}, x_{3}\right)$ is a ternary form, we let

$$
H(Q)=\frac{1}{2}\left[\begin{array}{ccc}
\frac{\partial^{2} Q}{\partial x_{1}^{2}} & \frac{\partial^{2} Q}{\partial x_{1} \partial x_{2}} & \frac{\partial^{2} Q}{\partial x_{1} \partial x_{3}} \\
\frac{\partial^{2} Q}{\partial x_{1} \partial x_{2}} & \frac{\partial^{2} Q}{\partial x_{2}^{2}} & \frac{\partial^{2} Q}{\partial x_{2} \partial x_{3}} \\
\frac{\partial^{2} Q}{\partial x_{1} \partial x_{3}} & \frac{\partial^{2} Q}{\partial x_{2} \partial x_{3}} & \frac{\partial^{2} Q}{\partial x_{3}^{2}}
\end{array}\right]
$$

be its Hessian matrix. For a quadratic form $Q$ over $K$, we let $H(Q)^{*}$ be the classical adjoint matrix of its Hessian $H(Q)$. Given two square matrices $A=\left(a_{i j}\right), B=\left(b_{i j}\right)$ of the same dimension, we also denote $\langle A, B\rangle=\sum_{i, j} a_{i j} b_{i j}$. 
With this notation, given a ternary quadric covariant $\boldsymbol{Q}\left(x_{1}, x_{2}, x_{3}\right)$ and a ternary quadric contravariant $\boldsymbol{\rho}\left(v_{1}, v_{2}, v_{3}\right)$, we let

$$
J_{11}(\boldsymbol{Q}, \boldsymbol{\rho})=\langle H(\boldsymbol{Q}), H(\boldsymbol{\rho})\rangle, \quad J_{22}(\boldsymbol{Q}, \boldsymbol{\rho})=\left\langle H(\boldsymbol{Q})^{*}, H(\boldsymbol{\rho})^{*}\right\rangle
$$

and

$$
J_{30}(\boldsymbol{Q})=\operatorname{det}(H(\boldsymbol{Q})), \quad J_{03}(\boldsymbol{\rho})=\operatorname{det}(H(\boldsymbol{\rho})) .
$$

Dixmier [9] and Ohno [28] (but see also [11]) have used these operators to determine new covariants, contravariants and invariants starting from the ternary quartic covariant $\boldsymbol{F}$, its Hessian $\boldsymbol{H}=216^{-1} \operatorname{det}(H(\boldsymbol{F}))$ and two contravariants $\boldsymbol{\sigma}$ (of degree 2 and order 4) and $\boldsymbol{\psi}$ (of degree 3 and order 6) that appear in [34, $\S 92, \S 292]$. First, they define a quadratic contravariant of degree 4

$$
\boldsymbol{\rho}=144^{-1} D(\boldsymbol{F}, \boldsymbol{\psi}) \text {. }
$$

From $\boldsymbol{\rho}$, one can derive two quadratic covariants of degree 5 (denoted $\tau$ and $\xi$ in the papers cited above)

$$
\boldsymbol{T}=144^{-1} D(\boldsymbol{F}, \boldsymbol{\rho}), \quad \boldsymbol{X}=72^{-1} D(\boldsymbol{H}, \boldsymbol{\sigma})
$$

and then two other quadratic contravariants, of degree 7 and 13 ,

$$
\boldsymbol{\eta}=12^{-1} D(\boldsymbol{X}, \boldsymbol{\rho}), \quad \chi=8^{-1} D(D(\boldsymbol{T}, \boldsymbol{\psi}), \boldsymbol{\psi})
$$

as well as a linear covariant of degree 14 (which is denoted by $\nu$ in the papers cite above), namely

$$
\boldsymbol{N}=8^{-1} D(D(\boldsymbol{H}, \boldsymbol{\rho}), \boldsymbol{\eta})
$$

The Dixmier invariants are then the algebraically independent invariants

$$
\begin{aligned}
& \boldsymbol{I}_{3}=144^{-1} D(\boldsymbol{F}, \boldsymbol{\sigma}), \quad \boldsymbol{I}_{9}=J_{11}(\boldsymbol{T}, \boldsymbol{\rho}), \quad \boldsymbol{I}_{15}=J_{30}(\boldsymbol{T}), \\
& \boldsymbol{I}_{6}=4608^{-1}\left(D(\boldsymbol{H}, \boldsymbol{\psi})-8 \boldsymbol{I}_{3}^{2}\right), \quad \boldsymbol{I}_{12}=J_{03}(\boldsymbol{\rho}), \quad \boldsymbol{I}_{18}=J_{22}(\boldsymbol{T}, \boldsymbol{\rho}) \\
& \text { and } \boldsymbol{I}_{27}=\operatorname{disc}(\boldsymbol{F}) \quad \text { (the discriminant of } \boldsymbol{F} \text { ). }
\end{aligned}
$$

To obtain the full ring of invariants of ternary quartics, we have to add the Ohno invariants

$$
\begin{array}{lll}
\boldsymbol{J}_{9}=J_{11}(\boldsymbol{X}, \boldsymbol{\rho}), & \boldsymbol{J}_{15}=J_{30}(\boldsymbol{X}), & \boldsymbol{I}_{21}=J_{03}(\boldsymbol{\eta}) \\
\boldsymbol{J}_{12}=J_{11}(\boldsymbol{T}, \boldsymbol{\eta}), & \boldsymbol{J}_{18}=J_{22}(\boldsymbol{X}, \boldsymbol{\rho}), & \boldsymbol{J}_{21}=J_{11}(\boldsymbol{N}, \boldsymbol{\eta})
\end{array}
$$

Theorem 1.6 (Ohno). The ring of invariants $K\left[\operatorname{Sym}^{4}\left(V^{*}\right)\right]^{\mathrm{SL}(V)}$ is generated by the 13 invariants (1.27) and (1.28).

The subscripts of the Dixmier-Ohno invariants indicate their degree in the coefficients of the universal form $\boldsymbol{F}$. In what follows, this is the degree to which we refer whenever we mention the degree of a homogeneous expression in the Dixmier-Ohno invariants.

Remark 1.7. The above theorem is originally due to Ohno [28]. Work by Elsenhans [11] gives a readable verification of his results. These invariants were further studied and implemented by Girard and Kohel in [15]. 
1.3. Joint invariants of octics and quartics. Let $W$ be a vector space of dimension 2 over $K$ with basis $w_{1}, w_{2}$ and corresponding dual basis $z_{1}, z_{2}$. We will now describe the basis of fundamental invariants calculated in [29] for the (diagonal) action of $\operatorname{SL}(W)$ on $\operatorname{Sym}^{8}\left(W^{*}\right) \oplus \operatorname{Sym}^{4}\left(W^{*}\right)$.

The Clebsch-Gordan decomposition [12] is the decomposition of $\mathrm{SL}(W)$-representations

$$
\operatorname{Sym}^{n_{1}}\left(W^{*}\right) \otimes \operatorname{Sym}^{n_{2}}\left(W^{*}\right) \simeq \bigoplus_{i=0}^{\min \left(n_{1}, n_{2}\right)} \operatorname{Sym}^{n_{1}+n_{2}-2 i}\left(W^{*}\right) .
$$

Let $i$ be an integer between 0 and $\min \left(n_{1}, n_{2}\right)$. Then we can consider the ClebschGordan projector (or transvectant)

$$
\operatorname{Sym}^{n_{1}}\left(W^{*}\right) \otimes \operatorname{Sym}^{n_{2}}\left(W^{*}\right) \longrightarrow \operatorname{Sym}^{n_{1}+n_{2}-2 i}\left(W^{*}\right), \quad b_{n_{1}} \otimes b_{n_{2}} \mapsto\left(b_{n_{1}}, b_{n_{2}}\right)_{i} .
$$

An explicit formula for the transvectant operator [30] can be obtained by using the bi-differential Cayley operator,

$$
\Omega_{y z}\left(b_{n_{1}}\left(y_{1}, y_{2}\right) \cdot b_{n_{2}}\left(z_{1}, z_{2}\right)\right):=\frac{\partial b_{n_{1}}}{y_{1}} \frac{\partial b_{n_{2}}}{z_{2}}-\frac{\partial b_{n_{1}}}{y_{2}} \frac{\partial b_{n_{2}}}{z_{1}}
$$

and the polarization operator

$$
\sigma_{y}\left(b_{n}\left(y_{1}, y_{2}\right)\right):=y_{1} \frac{\partial b_{n}}{\partial y_{1}}+y_{2} \frac{\partial b_{n}}{\partial y_{2}} .
$$

Let $i$ be as before. Then the transvectant of index $i$ is given by

$$
\left(b_{n_{1}}, b_{n_{2}}\right)_{i}:=\frac{\left(n_{1}-i\right) !}{n_{1} !} \frac{\left(n_{2}-i\right) !}{n_{2} !} \Omega_{y z}^{i} \sigma_{y}^{n_{1}-i} \sigma_{z}^{n_{2}-i}\left(b_{n_{1}}\left(y_{1}, y_{2}\right) \cdot b_{n_{2}}\left(z_{1}, z_{2}\right)\right) .
$$

Now an important result of classical invariant theory states that taking the closure of the universal pair $\left(\boldsymbol{b}_{n_{1}}, \boldsymbol{b}_{n_{2}}\right) \in \operatorname{Sym}^{n_{1}}\left(W^{*}\right) \times \operatorname{Sym}^{n_{2}}\left(W^{*}\right)$ under appropriate transvectant operations generates its covariant algebra [32]. Moreover, Gordan's algorithm enables one to construct a minimal basis of this algebra [16].

Using this algorithm in the case $n_{1}=8$ and $n_{2}=4$, Olive constructed Table 1 , where the 63 elements of the invariant basis have been overlaid in gray. We denote them by $\boldsymbol{j}_{d_{8}, d_{4}}$, sorting by bidegree as mentioned at the end of Section 1.1.

Two invariants in Olive's basis are pure invariants of $\boldsymbol{b}_{4}$ ( $c f$. Table 11.1), 9 of them are pure invariants of $\boldsymbol{b}_{8}(c f$. Table 11.2) and the 52 remaining are joint invariants ( $c f$. Table 11.3). From this table, we can for instance deduce that

$$
\begin{aligned}
& 2^{9} \cdot 3^{2} \cdot 5^{2} \cdot 7^{2} \times j_{1,2}=140 b_{4,4}^{2} b_{8,0}-35 b_{4,3} b_{4,4} b_{8,1}+ \\
&\left(10 b_{4,2} b_{4,4}+5 b_{4,3}^{2}\right) b_{8,2}-\left(5 b_{4,1} b_{4,4}+5 b_{4,2} b_{4,3}\right) b_{8,3}+ \\
&\left(4 b_{4,0} b_{4,4}+4 b_{4,1} b_{4,3}+2 b_{4,2}^{2}\right) b_{8,4}-\left(5 b_{4,0} b_{4,3}+5 b_{4,1} b_{4,2}\right) b_{8,5} \\
&+\left(10 b_{4,0} b_{4,2}+5 b_{4,1}^{2}\right) b_{8,6}-35 b_{4,0} b_{4,1} b_{8,7}+140 b_{4,0}^{2} b_{8,8} .
\end{aligned}
$$

Remark 1.8. Note that the invariant basis $\left\{\boldsymbol{j}_{2,0}, \boldsymbol{j}_{3,0}, \ldots \boldsymbol{j}_{10,0}\right\}$ of $\operatorname{Sym}^{8}\left(W^{*}\right)$ given in Table 11.2 differs slightly from the basis $\left\{\boldsymbol{J}_{2}, \boldsymbol{J}_{3}, \ldots, \boldsymbol{J}_{10}\right\}$ of [35]. We have

$$
\begin{aligned}
\boldsymbol{J}_{2}=2^{7} \cdot 3^{2} \cdot 5 \cdot 7 \boldsymbol{j}_{2,0}, \boldsymbol{J}_{3}=2^{10} \cdot 3^{3} \cdot 5 \cdot 7 & \boldsymbol{j}_{3,0}, \\
\boldsymbol{J}_{4} & =2^{13} \cdot 3^{3} \cdot 5^{2}\left(\begin{array}{ll}
50 \boldsymbol{j}_{2,0}^{2}-33 \boldsymbol{j}_{4,0}
\end{array}\right)
\end{aligned}
$$

and similar equations exist for the other $\boldsymbol{J}_{d}$. 
TABle 1. A minimal basis of 63 invariants for $\operatorname{Sym}^{8}\left(W^{*}\right) \oplus \operatorname{Sym}^{4}\left(W^{*}\right)$.

$$
\begin{gathered}
\begin{array}{cc|c|c|c|c|}
\text { Covariant }_{(d, o)} & \text { Invariant } & \text { Covariant } & (d, o) & \text { Invariant } & \text { Covariant } \\
\hline \hline \boldsymbol{g}_{1}=\boldsymbol{b}_{4} & (1,4) & \boldsymbol{j}_{0,2}=\left(\boldsymbol{g}_{1}, \boldsymbol{g}_{1}\right)_{4} & \boldsymbol{g}_{2}=\left(\boldsymbol{g}_{1}, \boldsymbol{g}_{1}\right)_{2}(2,4) & \boldsymbol{j}_{0,3}=\left(\boldsymbol{g}_{2}, \boldsymbol{g}_{1}\right)_{4} & \mid \boldsymbol{g}_{3}=\left(\boldsymbol{g}_{2}, \boldsymbol{g}_{1}\right)_{2}(3,6)
\end{array} \\
\text { 1.1: Covariants of } \operatorname{Sym}^{4}\left(W^{*}\right) .
\end{gathered}
$$

\begin{tabular}{|c|c|c|c|c|c|c|c|}
\hline Covariant & $(d, o)$ & Covariant & $(d, o)$ & Covariant & $(d, o)$ & Covariant & \\
\hline $\boldsymbol{f}_{1}=\boldsymbol{b}_{8}$ & $(1,8)$ & $\boldsymbol{f}_{19}=\left(\boldsymbol{f}_{13}\right.$ & $(4,10)$ & $\boldsymbol{f}_{37}=\left(\boldsymbol{f}_{33}, \boldsymbol{f}_{1}\right)_{7}$ & $(6,4)$ & $\boldsymbol{f}_{55}=\left(\boldsymbol{f}_{51}, \boldsymbol{f}_{1}\right)_{5}$ & $(8,4$ \\
\hline $\boldsymbol{j}_{2,0}=\left(\boldsymbol{f}_{1}, \boldsymbol{f}_{1}\right)_{8}$ & & $\boldsymbol{f}_{20}=\left(\boldsymbol{f}_{12}, \boldsymbol{f}_{1}\right)_{6}$ & $(4,10$ & $\boldsymbol{f}_{38}=\left(\boldsymbol{f}_{32}, \boldsymbol{f}_{1}\right)_{7}$ & $(6,4)$ & $\boldsymbol{f}_{56}=\left(\boldsymbol{f}_{50}, \boldsymbol{f}_{1}\right)_{5}$ & 8,4 \\
\hline $\boldsymbol{f}_{3}=\left(\boldsymbol{f}_{1}, \boldsymbol{f}\right.$ & $(2,4)$ & $\boldsymbol{f}_{21}=\left(\boldsymbol{f}_{13}, \boldsymbol{f}_{1}\right)_{7}$ & $(4,12)$ & $\boldsymbol{f}_{39}=\left(\boldsymbol{f}_{34}, \boldsymbol{f}_{1}\right)_{8}$ & $(6,6)$ & $\boldsymbol{f}_{57}=\left(\boldsymbol{f}_{51}, \boldsymbol{f}_{1}\right)_{4}$ & $(8,6)$ \\
\hline $\boldsymbol{f}_{4}=\left(\boldsymbol{f}_{1}, \boldsymbol{f}\right.$ & $(2,8)$ & $\boldsymbol{f}_{22}=\left(\boldsymbol{f}_{13}, \boldsymbol{f}_{1}\right)_{6}$ & $(4,14)$ & $\boldsymbol{f}_{40}=\left(\boldsymbol{f}_{33}, \boldsymbol{f}_{1}\right)_{6}$ & $(6,6)$ & $\boldsymbol{f}_{58}=\left(\boldsymbol{f}_{50}, \boldsymbol{f}_{1}\right)_{4}$ & $(8,6)$ \\
\hline $\boldsymbol{f}_{5}=\left(\boldsymbol{f}_{1}, \boldsymbol{f}_{1}\right)_{2}$ & $(2,12)$ & $\boldsymbol{f}_{23}=\left(\boldsymbol{f}_{13}, \boldsymbol{f}_{1}\right)_{4}$ & $(4,18)$ & $\boldsymbol{f}_{41}=\left(\boldsymbol{f}_{32}, \boldsymbol{f}_{1}\right)_{6}$ & $(6,6)$ & $\boldsymbol{j}_{9,0}=\left(\boldsymbol{f}_{15} \boldsymbol{f}_{16}, \boldsymbol{f}_{1}\right)_{8}$ & \\
\hline $\boldsymbol{j}_{3,0}=\left(\boldsymbol{f}_{4}, \boldsymbol{f}_{1}\right)_{8}$ & & $\boldsymbol{j}_{5,0}=\left(\boldsymbol{f}_{3}^{2}, \boldsymbol{f}_{1}\right)_{8}$ & & $\boldsymbol{f}_{42}=\left(\boldsymbol{f}_{34}, \boldsymbol{f}_{1}\right)_{7}$ & $(6,8)$ & $\boldsymbol{f}_{60}=\left(\boldsymbol{f}_{58}, \boldsymbol{f}_{1}\right)_{6}$ & $(9,2)$ \\
\hline $\boldsymbol{f}_{7}=\left(\boldsymbol{f}_{5}, \boldsymbol{f}_{1}\right)_{8}$ & $(3,4)$ & $\boldsymbol{f}_{25}=\left(\boldsymbol{f}_{20}, \boldsymbol{f}_{1}\right)_{8}$ & $(5,2)$ & $\boldsymbol{f}_{43}=\left(\boldsymbol{f}_{34}, \boldsymbol{f}_{1}\right)_{6}$ & $(6,10)$ & $\boldsymbol{f}_{61}=\left(\boldsymbol{f}_{5}\right.$ & $(9,2)$ \\
\hline $\boldsymbol{f}_{8}=\left(\boldsymbol{f}_{5}\right.$, & $(3,6)$ & $\boldsymbol{f}_{26}=\left(\boldsymbol{f}_{21}, \boldsymbol{f}_{1}\right)_{8}$ & $(5,4)$ & $\boldsymbol{j}_{7,0}=\left(\boldsymbol{f}_{7}^{2}, \boldsymbol{f}_{1}\right)_{8}$ & & $\boldsymbol{f}_{62}=\left(\boldsymbol{f}_{16} \boldsymbol{f}_{17}, \boldsymbol{f}_{1}\right)_{8}$ & $(9,2)$ \\
\hline $\boldsymbol{f}_{9}=\left(\boldsymbol{f}_{5}\right.$ & $(3,8)$ & $\boldsymbol{f}_{27}=\left(\boldsymbol{f}_{20}, \boldsymbol{f}_{1}\right)_{7}$ & $(5,4)$ & $\boldsymbol{f}_{45}=\left(\boldsymbol{f}_{4}\right.$ & $(7,2)$ & $\boldsymbol{f}_{63}=\left(\boldsymbol{f}_{58}, \boldsymbol{f}_{1}\right)_{5}$ & $(9,4)$ \\
\hline $\boldsymbol{f}_{10}=\left(\boldsymbol{f}_{5}, \boldsymbol{f}_{1}\right)_{5}$ & $(3,10)$ & $\boldsymbol{f}_{28}=\left(\boldsymbol{f}_{22}, \boldsymbol{f}_{1}\right)_{8}$ & $(5,6)$ & $\boldsymbol{f}_{46}=\left(\boldsymbol{f}_{4}\right.$ & $(7,2)$ & $\boldsymbol{j}_{10,0}=\left(\boldsymbol{f}_{17} \boldsymbol{f}_{25}, \boldsymbol{f}_{1}\right)_{8}$ & \\
\hline $\boldsymbol{f}_{11}=\left(\boldsymbol{f}_{5}\right.$ & $(3,12$ & $\boldsymbol{f}_{29}=\left(\boldsymbol{f}_{2}\right.$ & $(5,6)$ & $\boldsymbol{f}_{47}=\left(\boldsymbol{f}_{4}\right.$ & $(7,4)$ & $\boldsymbol{f}_{65}=\left(\boldsymbol{f}_{17} \boldsymbol{f}_{27}, \boldsymbol{f}_{1}\right)_{8}$ & $(10,2$ \\
\hline $\boldsymbol{f}_{12}=(\boldsymbol{f}$ & $(3,1)$ & $\boldsymbol{f}_{30}=\left(\boldsymbol{f}_{2}\right.$ & $(5$, & $\boldsymbol{f}_{48}=($ & $(7$, & $\boldsymbol{f}_{66}=\left(\boldsymbol{f}_{1}\right.$ & $(10,2$ \\
\hline $\boldsymbol{f}_{13}=(\boldsymbol{f}$ & $(3,18$ & $\boldsymbol{f}_{31}=\left(\boldsymbol{f}_{2}\right.$ & $(5,10$ & $\boldsymbol{f}_{49}=($ & $(7$, & $\boldsymbol{f}_{67}=\left(\boldsymbol{f}_{27} \boldsymbol{f}_{29}, \boldsymbol{f}_{1}\right)_{8}$ & $(11,2)$ \\
\hline $\boldsymbol{j}_{4,0}=\left(\boldsymbol{f}_{9}\right.$, & & $\boldsymbol{f}_{32}=\left(\boldsymbol{f}_{2}\right.$ & $(5,10$ & $\boldsymbol{f}_{50}=(\boldsymbol{f}$ & $(7$, & $\boldsymbol{f}_{68}=\left(\boldsymbol{f}_{27} \boldsymbol{f}_{28}, \boldsymbol{f}_{1}\right)_{8}$ & $(11,2)$ \\
\hline$f_{15}=$ & $(4,4)$ & $\boldsymbol{f}_{33}=\left(\boldsymbol{f}_{21}, \boldsymbol{f}_{1}\right)_{5}$ & $(5,10)$ & $\boldsymbol{f}_{51}=\left(\boldsymbol{f}_{41}, \boldsymbol{f}_{1}\right)_{4}$ & $(7,6)$ & $\boldsymbol{f}_{69}=\left(\boldsymbol{f}_{29} \boldsymbol{f}_{38}, \boldsymbol{f}_{1}\right)_{8}$ & $(12,2)$ \\
\hline$f_{16}=$ & $(4,4)$ & $\boldsymbol{f}_{34}=\left(\boldsymbol{f}_{23}, \boldsymbol{f}_{1}\right)_{6}$ & $(5,14$ & $\boldsymbol{j}_{8,0}=\left(\boldsymbol{f}_{7} \boldsymbol{f}_{16}, \boldsymbol{f}_{1}\right)_{8}$ & & & \\
\hline $\boldsymbol{f}_{17}$ & $(4,6$ & $\boldsymbol{j}_{6,0}=\left(\boldsymbol{f}_{3} \boldsymbol{f}_{7}, \boldsymbol{f}_{1}\right)_{8}$ & & $\boldsymbol{f}_{53}=\left(\boldsymbol{f}_{5}\right.$ & $(8$, & & \\
\hline $\boldsymbol{f}_{18}=\left(\boldsymbol{f}_{12}, \boldsymbol{f}_{1}\right)_{7}$ & $(4,8$ & $\boldsymbol{f}_{36}=\left(\boldsymbol{f}_{33}, \boldsymbol{f}_{1}\right)_{8}$ & $(6,2)$ & $\boldsymbol{f}_{54}=\left(\boldsymbol{f}_{50}, \boldsymbol{f}_{1}\right)_{6}$ & $(8,2)$ & & \\
\hline
\end{tabular}

\begin{tabular}{|c|c|c|c|}
\hline Invariant & Invariant & Invariant & Invariant \\
\hline$\overline{\boldsymbol{j}_{1,2}=\left(\boldsymbol{f}_{1}, \boldsymbol{g}_{1}^{2}\right)_{8}}$ & $\overline{\boldsymbol{j}_{2,4}=\left(\boldsymbol{f}_{4}, \boldsymbol{g}_{2}^{2}\right)_{8}}$ & $\boldsymbol{j}_{4,3}=\left(\boldsymbol{f}_{18}, \boldsymbol{g}_{1} \boldsymbol{g}_{2}\right)_{8}$ & $\overline{\boldsymbol{j}_{5,3}=\left(\boldsymbol{f}_{29}, \boldsymbol{g}_{3}\right)_{6}}$ \\
\hline $\boldsymbol{j}_{2,1}=\left(\boldsymbol{f}_{3}, \boldsymbol{g}_{1}\right)_{4}$ & $\boldsymbol{j}_{2,4}^{\prime}=\left(\boldsymbol{f}_{5}, \boldsymbol{g}_{1}^{2} \boldsymbol{g}_{2}\right)_{12}$ & $\boldsymbol{j}_{4,3}^{\prime}=\left(\boldsymbol{f}_{17}, \boldsymbol{g}_{3}\right)_{6}$ & $\boldsymbol{j}_{5,3}^{\prime}=\left(\boldsymbol{f}_{30}, \boldsymbol{g}_{1} \boldsymbol{g}_{2}\right)_{8}$ \\
\hline $\boldsymbol{j}_{1,3}=\left(\boldsymbol{f}_{1}, \boldsymbol{g}_{1} \boldsymbol{g}_{2}\right)_{8}$ & $\boldsymbol{j}_{3,3}=\left(\boldsymbol{f}_{11}, \boldsymbol{g}_{1}^{3}\right)_{12}$ & $\boldsymbol{j}_{4,3}^{\prime \prime}=\left(\boldsymbol{f}_{21}, \boldsymbol{g}_{1}^{3}\right)_{12}$ & $\boldsymbol{j}_{6,2}=\left(\boldsymbol{f}_{37}, \boldsymbol{g}_{2}\right)_{4}$ \\
\hline $\boldsymbol{j}_{2,2}=\left(\boldsymbol{f}_{4}, \boldsymbol{g}_{1}^{2}\right)_{8}$ & $\boldsymbol{j}_{3,3}^{\prime}=\left(\boldsymbol{f}_{9}, \boldsymbol{g}_{1} \boldsymbol{g}_{2}\right)_{8}$ & $\boldsymbol{j}_{5,2}=\left(\boldsymbol{f}_{30}, \boldsymbol{g}_{1}^{2}\right)_{8}$ & $\boldsymbol{j}_{6,2}^{\prime}=\left(\boldsymbol{f}_{38}, \boldsymbol{g}_{2}\right)_{4}$ \\
\hline $\boldsymbol{j}_{2,2}^{\prime}=\left(\boldsymbol{f}_{3}, \boldsymbol{g}_{2}\right)_{4}$ & $\boldsymbol{j}_{3,3}^{\prime \prime}=\left(\boldsymbol{f}_{8}, \boldsymbol{g}_{3}\right)_{6}$ & $\boldsymbol{j}_{5,2}^{\prime}=\left(\boldsymbol{f}_{27}, \boldsymbol{g}_{2}\right)_{4}$ & $\boldsymbol{j}_{6,2}^{\prime \prime}=\left(\boldsymbol{f}_{42}, \boldsymbol{g}_{1}^{2}\right)_{8}$ \\
\hline $\boldsymbol{j}_{3,1}=\left(\boldsymbol{f}_{7}, \boldsymbol{g}_{1}\right)_{4}$ & $\boldsymbol{j}_{4,2}=\left(\boldsymbol{f}_{15}, \boldsymbol{g}_{2}\right)_{4}$ & $\boldsymbol{j}_{5,2}^{\prime \prime}=\left(\boldsymbol{f}_{26}, \boldsymbol{g}_{2}\right)_{4}$ & $\boldsymbol{j}_{6,3}=\left(\boldsymbol{f}_{8}^{2}, \boldsymbol{g}_{1}^{3}\right)_{12}$ \\
\hline $\boldsymbol{j}_{1,4}=\left(\boldsymbol{f}_{1}, \boldsymbol{g}_{2}^{2}\right)_{8}$ & $\boldsymbol{j}_{4,2}^{\prime}=\left(\boldsymbol{f}_{18}, \boldsymbol{g}_{1}^{2}\right)_{8}$ & $\boldsymbol{j}_{6,1}=\left(\boldsymbol{f}_{37}, \boldsymbol{g}_{1}\right)_{4}$ & $\boldsymbol{j}_{7,2}=\left(\boldsymbol{f}_{48}, \boldsymbol{g}_{2}\right)_{4}$ \\
\hline $\boldsymbol{j}_{2,3}=\left(\boldsymbol{f}_{4}, \boldsymbol{g}_{1} \boldsymbol{g}_{2}\right)_{8}$ & $\boldsymbol{j}_{4,2}^{\prime \prime}=\left(\boldsymbol{f}_{16}, \boldsymbol{g}_{2}\right)_{4}$ & $\boldsymbol{j}_{6,1}^{\prime}=\left(\boldsymbol{f}_{38}, \boldsymbol{g}_{1}\right)_{4}$ & $\boldsymbol{j}_{7,2}^{\prime}=\left(\boldsymbol{f}_{47}, \boldsymbol{g}_{2}\right)_{4}$ \\
\hline $\boldsymbol{j}_{2,3}^{\prime}=\left(\boldsymbol{f}_{5}, \boldsymbol{g}_{1}^{3}\right)_{12}$ & $\boldsymbol{j}_{5,1}=\left(\boldsymbol{f}_{26}, \boldsymbol{g}_{1}\right)_{4}$ & $\boldsymbol{j}_{7,1}=\left(\boldsymbol{f}_{47}, \boldsymbol{g}_{1}\right)_{4}$ & $\boldsymbol{j}_{8,1}=\left(\boldsymbol{f}_{55}, \boldsymbol{g}_{1}\right)_{4}$ \\
\hline $\boldsymbol{j}_{3,2}=\left(\boldsymbol{f}_{7}, \boldsymbol{g}_{2}\right)_{4}$ & $\boldsymbol{j}_{5,1}^{\prime}=\left(\boldsymbol{f}_{27}, \boldsymbol{g}_{1}\right)_{4}$ & $\boldsymbol{j}_{7,1}^{\prime}=\left(\boldsymbol{f}_{48}, \boldsymbol{g}_{1}\right)_{4}$ & $\boldsymbol{j}_{8,1}^{\prime}=\left(\boldsymbol{f}_{56}, \boldsymbol{g}_{1}\right)_{4}$ \\
\hline $\boldsymbol{j}_{3,2}^{\prime}=\left(\boldsymbol{f}_{9}, \boldsymbol{g}_{1}^{2}\right)_{8}$ & $\boldsymbol{j}_{2,5}=\left(\boldsymbol{f}_{5}, \boldsymbol{g}_{1} \boldsymbol{g}_{2}^{2}\right)_{12}$ & $\boldsymbol{j}_{3,5}=\left(\boldsymbol{f}_{11}, \boldsymbol{g}_{1} \boldsymbol{g}_{2}^{2}\right)_{12}$ & $\boldsymbol{j}_{8,2}=\left(\boldsymbol{f}_{56}, \boldsymbol{g}_{2}\right)_{4}$ \\
\hline $\boldsymbol{j}_{4,1}=\left(\boldsymbol{f}_{15}, \boldsymbol{g}_{1}\right)_{4}$ & $\boldsymbol{j}_{3,4}=\left(\boldsymbol{f}_{10}, \boldsymbol{g}_{1} \boldsymbol{g}_{3}\right)_{10}$ & $\boldsymbol{j}_{4,4}=\left(\boldsymbol{f}_{20}, \boldsymbol{g}_{1} \boldsymbol{g}_{3}\right)_{10}$ & $\boldsymbol{j}_{9,1}=\left(\boldsymbol{f}_{63}, \boldsymbol{g}_{1}\right)_{4}$ \\
\hline $\boldsymbol{j}_{4,1}^{\prime}=\left(\boldsymbol{f}_{16}, \boldsymbol{g}_{1}\right)_{4}$ & $\boldsymbol{j}_{3,4}^{\prime}=\left(\boldsymbol{f}_{11}, \boldsymbol{g}_{1}^{2} \boldsymbol{g}_{2}\right)_{12}$ & $\boldsymbol{j}_{4,4}^{\prime}=\left(\boldsymbol{f}_{21}, \boldsymbol{g}_{1}^{2} \boldsymbol{g}_{2}\right)_{12}$ & $\boldsymbol{j}_{10,1}=\left(\boldsymbol{f}_{25}^{2}, \boldsymbol{g}_{1}\right)_{4}$ \\
\hline
\end{tabular}

1.2: Covariants of $\operatorname{Sym}^{8}\left(W^{*}\right)$.

1.3: Joint invariants

\section{Sections And Rationality}

2.1. A result by Van Rijnswou. In this section we recall an explicit isomorphism of SL(W)-representations that was first constructed in [40]. For reasons that we mentioned in the introduction it has proved to be an extremely fruitful inroad into our problem.

Let $W$ be a vector space of dimension 2 over $K$ with basis $w_{1}, w_{2}$ and corresponding dual basis $z_{1}, z_{2}$. Consider the vector space $V=\operatorname{Sym}^{2}(W)$, which is of dimension 3. We fix the basis $v_{1}=w_{1}^{2}, v_{2}=2 w_{1} w_{2}, v_{3}=w_{2}^{2}$ of $V$, with corresponding dual basis $x_{1}=z_{1}^{2}, x_{2}=\frac{1}{2} z_{1} z_{2}, x_{3}=z_{2}^{2}$. The induced left action of $\mathrm{SL}(W)$ on 
$V$ in the bases chosen above is described by the homomorphism

$$
\begin{aligned}
h: \mathrm{SL}(W) & \rightarrow \mathrm{SL}\left(\mathrm{Sym}^{2}(W)\right)=\mathrm{SL}(V) \\
\left(\begin{array}{ll}
a & b \\
c & d
\end{array}\right) & \mapsto\left(\begin{array}{ccc}
a^{2} & 2 a b & b^{2} \\
a c & a d+b c & b d \\
c^{2} & 2 c d & d^{2}
\end{array}\right)
\end{aligned}
$$

since for example $w_{1}^{2}$ is sent to $\left(a w_{1}+c w_{2}\right)^{2}=a^{2} w_{1}^{2}+a c\left(2 w_{1} w_{2}\right)+c^{2} w_{2}^{2}=a^{2} v_{1}+$ $a c v_{2}+c^{2} v_{3}$.

It is known that the substitution action of $\mathrm{SL}(W)$ on the space of binary forms $\operatorname{Sym}^{2}\left(W^{*}\right)$ is orthonormal with respect to the discriminant form. So too is the action on $\operatorname{Sym}^{2}(W)$ above orthogonal with respect to the dual of this discriminant form, which we calculate to be

$$
\left(z_{1} z_{2}\right)^{2}-4 z_{1}^{2} z_{2}^{2}=4\left(x_{2}^{2}-x_{1} x_{3}\right) .
$$

In other words, the homomorphism (2.1) has image in $\mathrm{SO}\left(x_{2}^{2}-x_{1} x_{3}\right)$. Because its tangent map is surjective, it is itself surjective, as $\mathrm{SO}\left(x_{2}^{2}-x_{1} x_{3}\right)$ is irreducible. Though we will not use this fact, we mention that its kernel has order 2.

Similarly the homomorphism

$$
\begin{aligned}
h^{*}: \mathrm{SL}(W) & \rightarrow \mathrm{SL}\left(\operatorname{Sym}^{2}(W)\right)=\mathrm{SL}(V) \\
\left(\begin{array}{ll}
a & b \\
c & d
\end{array}\right) & \mapsto\left(\begin{array}{ccc}
a^{2} & a b & b^{2} \\
2 a c & a d+b c & 2 b d \\
c^{2} & c d & d^{2}
\end{array}\right)
\end{aligned}
$$

is a degree 2 surjection onto $\mathrm{SO}\left(v_{2}^{2}-v_{1} v_{3}\right)$. Note that the notation $h^{*}$ is slightly abusive here, because it is not $h$ itself that is dualized; rather, as a subgroup of $\mathrm{SL}(V)$ the image of $h$ fixes the element $x_{2}^{2}-x_{1} x_{3}$ while $h^{*}$ fixes its dual $v_{2}^{2}-v_{1} v_{3}$.

The irreducible representations of $\mathrm{SL}(W)$ are up to isomorphism described by the symmetric powers $\operatorname{Sym}^{d}\left(W^{*}\right)$ by [36, Proposition 3.2.1]. By [10, Theorem 5.4], there exists a decomposition

$$
\operatorname{Sym}^{4}\left(\operatorname{Sym}^{2}\left(W^{*}\right)\right) \cong \operatorname{Sym}^{8}\left(W^{*}\right) \oplus \operatorname{Sym}^{4}\left(W^{*}\right) \oplus \operatorname{Sym}^{0}\left(W^{*}\right)
$$

of SL $(W)$-representations. Following [40], we will now indicate how to calculate an explicit isomorphism (2.4). We focus on the covariant case to fix ideas, so that we use the homomorphism $h$; in the contravariant case one works with $h^{*}$ instead.

Consider the classical generators

$$
\mathfrak{e}=\left(\begin{array}{ll}
0 & 1 \\
0 & 0
\end{array}\right), \mathfrak{h}=\left(\begin{array}{cc}
1 & 0 \\
0 & -1
\end{array}\right), \mathfrak{f}=\left(\begin{array}{ll}
0 & 0 \\
1 & 0
\end{array}\right)
$$

of the Lie algebra $\mathfrak{s l}(W)$ of $\mathrm{SL}(W)$, which acts on the right on both sides of (2.4) by taking the differential of the action of the latter group. For example, the oneparameter subgroup of $\mathrm{SL}(W)$ given by

$$
\left(\begin{array}{ll}
1 & \varepsilon \\
0 & 1
\end{array}\right)
$$

gives rise to $\mathfrak{e}$ upon deriving with respect to the parameter $\varepsilon$ at $\varepsilon=0$. The action of the matrix (2.6) sends $\left(z_{1}, z_{2}\right)$ to $\left(z_{1}+\varepsilon z_{2}, z_{2}\right)$. Deriving the latter expression with respect to $\varepsilon$ at $\varepsilon=0$ we obtain $\left(z_{2}, 0\right)$, which indeed equals $\left(z_{1}, z_{2}\right) \cdot \mathfrak{e}$.

The right action on $\operatorname{Sym}^{d}\left(W^{*}\right)$ is derived from (2.5) by the Leibniz rule; $\mathfrak{e}$ sends a binary form $b$ to $z_{1} \cdot \mathfrak{e} \frac{\partial b}{\partial z_{1}}+z_{2} \cdot \mathfrak{e} \frac{\partial b}{\partial z_{2}}=z_{2} \frac{\partial b}{\partial z_{1}}$, and similarly for the other generators. 
Taking the derivative at the identity of the map $h$ in (2.1), we obtain the action on $V$, which is represented by the matrices

$$
(D h) \mathfrak{e}=\left(\begin{array}{ccc}
0 & 2 & 0 \\
0 & 0 & 1 \\
0 & 0 & 0
\end{array}\right),(D h) \mathfrak{h}=\left(\begin{array}{ccc}
2 & 0 & 0 \\
0 & 0 & 0 \\
0 & 0 & -2
\end{array}\right),(D h) \mathfrak{f}=\left(\begin{array}{ccc}
0 & 0 & 0 \\
1 & 0 & 0 \\
0 & 2 & 0
\end{array}\right)
$$

To verify the equalities in (2.7), we can also employ a more direct argument. For example, via $h$ the action of the matrices (2.6) transforms into that of

$$
\left(\begin{array}{ccc}
1 & 2 \varepsilon & \varepsilon^{2} \\
0 & 1 & \varepsilon \\
0 & 0 & 1
\end{array}\right)
$$

and indeed upon deriving with respect to $\varepsilon$ at $\varepsilon=0$ we obtain the first matrix in (2.7).

In what follows, we consider $V$ as an $\mathrm{SL}(W)$-representation via (2.7), so that the left action of $\mathfrak{e}($ resp. $\mathfrak{h}, \mathfrak{f})$ is represented by the matrix $(D h) \mathfrak{e}(\operatorname{resp} .(D h) \mathfrak{h},(D h) \mathfrak{f})$ in (2.7) instead. Using the Leibniz rule we see can once more derive the right action on $\operatorname{Sym}^{4}\left(V^{*}\right)$ : for example, the action of $\mathfrak{e}$ sends a ternary quartic form $F$ to

$$
2 x_{2} \frac{\partial F}{\partial x_{1}}+x_{3} \frac{\partial F}{\partial x_{2}} .
$$

We now use the theory of highest weight vectors on these representations of $\mathfrak{s t}(W)$. In a nutshell, the special case of this theory for representations of $\mathfrak{s l}(W)$ (concerning which a fuller account is available in [12]) amounts to the following observation. For the standard representations $\operatorname{Sym}^{p}\left(W^{*}\right)$ of $\mathrm{SL}(W)$ we observe that there is up to scaling a unique vector $u$ whose weight for the action of $\mathfrak{h}$ is the highest possible, namely $u=z_{1}^{p}$. This vector also has the property that $u \cdot \mathfrak{f}=0$, and moreover the complete representation is spanned by the $p+1$ powers $\left\{u \cdot \mathfrak{e}^{(0)}, u \cdot \mathfrak{e}^{(1)}, \ldots, u \cdot \mathfrak{e}^{(p)}\right\}$, which indeed are up to scaling the vectors $\left\{z_{1}^{p}, z_{1}^{p-1} z_{2}, \ldots, z_{2}^{p}\right\}$.

To decompose an arbitrary finite-dimensional representation $U$ of $\mathfrak{s l}(W)$ into its irreducible constituents, we first track down a basis of the subspace whose elements are of highest weight $\lambda_{0}$ under the action of $\mathfrak{h}$. We know by the above that their images under $\mathfrak{e}$ generate a subrepresentation that is isomorphic to a power $\operatorname{Sym}^{p_{0}}\left(W^{*}\right)$, the multiplicity being given by the dimension of the subspace. Subsequently, we determine the subspace of all vectors $u \in U$ that are of the next highest weight $\lambda_{1}$ under $\mathfrak{h}$ but not yet in the image of the subrepresentations found and that additionally satisfy $u \cdot \mathfrak{f}=0$ (a condition satisfied automatically for the vectors of highest weight $\lambda_{0}$ ). The space of these vectors gives rise to another subrepresentation with multiplicity as above. Continuing in this way, we obtain our decomposition.

In our case we obtain that a subrepresentation of the left hand side of (2.4) that is isomorphic to $\operatorname{Sym}^{8}\left(W^{*}\right)$ is generated by the successive images under $\mathfrak{e}$ the vector of highest weight $x_{1}^{4}$, which is unique up to a scalar multiple. We map this vector to $z_{1}^{8}$, the vector of highest weight in $\operatorname{Sym}^{8}\left(W^{*}\right)$, which then forces our hand by equivariance.

Another subrepresentation, isomorphic to $\operatorname{Sym}^{4}\left(W^{*}\right)$, can be found by considering a vector of weight 4 that is annihilated by $\mathfrak{f}$. This is $x_{1}^{3} x_{3}-x_{1}^{2} x_{2}^{2}$ or one of its multiples. We map this vector to $z_{1}^{4}$, the vector of highest weight in $\operatorname{Sym}^{4}\left(W^{*}\right)$, 
and again our hand is forced. Finally, a new vector of weight 0 that is annihilated by $\mathfrak{f}$ is given by $x_{1}^{2} x_{3}^{2}-2 x_{1} x_{2}^{2} x_{3}+x_{2}^{4}$ or one of its multiples. We map this to $1 \in \operatorname{Sym}^{0}\left(W^{*}\right)$, and we finally have an equivariant isomorphism (2.4) for the homomorphism $h$. After proceeding similarly for the dual version $h^{*}$ from (2.3), we have two equivariant linear maps

$$
\begin{aligned}
\ell, \ell^{*}: \operatorname{Sym}^{4}\left(V^{*}\right) & \rightarrow \operatorname{Sym}^{8}\left(W^{*}\right) \oplus \operatorname{Sym}^{4}\left(W^{*}\right) \oplus \operatorname{Sym}^{0}\left(W^{*}\right), \\
F & \mapsto\left(b_{8}, b_{4}, b_{0}\right),
\end{aligned}
$$

and our construction shows that the following theorem holds.

Theorem 2.1 (Van Rijnswou, 2001 [40]). We have

$$
\ell(F \cdot h(T))=\ell(F) \cdot T
$$

and

$$
\ell^{*}\left(F \cdot h^{*}(T)\right)=\ell^{*}(F) \cdot T .
$$

In other words, $\ell,\left(\right.$ resp. $\left.\ell^{*}\right)$ gives an $\mathrm{SL}(W)$-equivariant linear map

$$
\operatorname{Sym}^{4}\left(\operatorname{Sym}^{2}\left(W^{*}\right)\right) \rightarrow \operatorname{Sym}^{8}\left(W^{*}\right) \oplus \operatorname{Sym}^{4}\left(W^{*}\right) \oplus \operatorname{Sym}^{0}(W *)
$$

when $\operatorname{Sym}^{4}\left(\operatorname{Sym}^{2}\left(W^{*}\right)\right)$ is considered as an $\mathrm{SL}(W)$-representation via $h$ (resp. $\left.h^{*}\right)$.

Note that the maps $\ell$ and $\ell^{*}$ are not unique, but in light of Schur's Lemma (or the explicit construction above) they are up to a scalar multiple per irreducible component.

In coordinates, we can describe these maps as follows. Let a basis of the left-hand side of (2.10) be given by

$$
x_{1}^{4}, x_{1}^{3} x_{2}, x_{1}^{3} x_{3}, x_{1}^{2} x_{2}^{2}, x_{1}^{2} x_{2} x_{3}, x_{1}^{2} x_{3}^{2}, x_{1} x_{2}^{3}, x_{1} x_{2}^{2} x_{3}, x_{1} x_{2} x_{3}^{2}, x_{1} x_{3}^{3}, x_{2}^{4}, x_{2}^{3} x_{3}, x_{2}^{2} x_{3}^{2}, x_{2} x_{3}^{3}, x_{3}^{4}
$$

and let a basis for the right-hand side be given by

$$
z_{1}^{8}, z_{1}^{7} z_{2}, z_{1}^{6} z_{2}^{2}, z_{1}^{5} z_{2}^{3}, z_{1}^{4} z_{2}^{4}, z_{1}^{3} z_{2}^{5}, z_{1}^{2} z_{2}^{6}, z_{1} z_{2}^{7}, z_{2}^{8}, \quad z_{1}^{4}, z_{1}^{3} z_{2}, z_{1}^{2} z_{2}^{2}, z_{1} z_{2}^{3}, z_{2}^{4}, \quad 1
$$

Then with respect to these bases, $\ell$ is given by the right action of

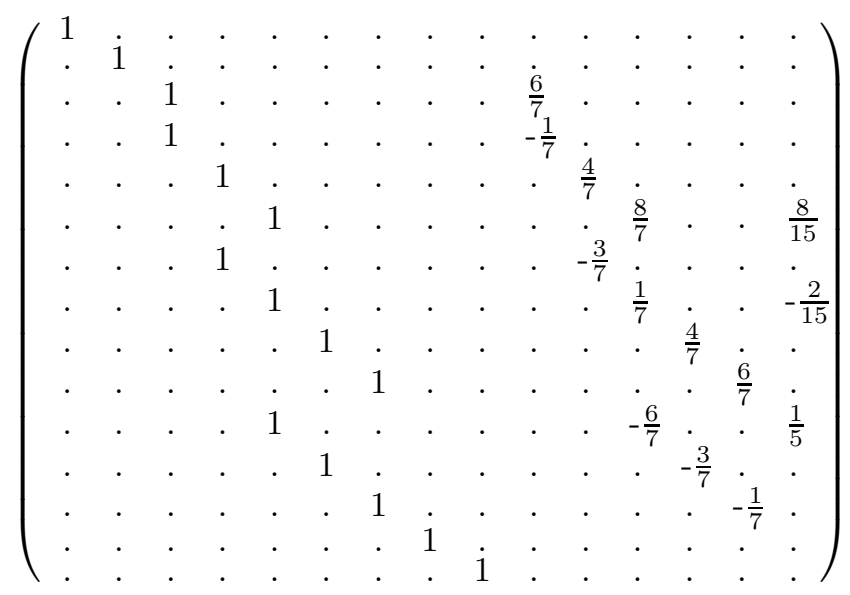


whereas $\ell^{*}$ is given by the right action of

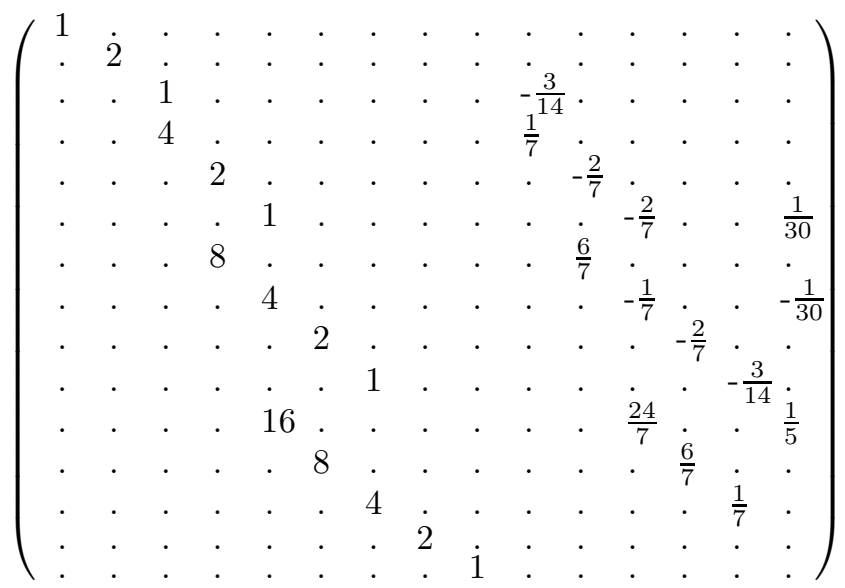

where we have replaced zeros with dots for clarity.

Remark 2.2. One can explicitly verify that the linear maps $\ell, \ell^{*}$ defined by the matrices (2.16) and (2.17) still define equivariant maps in the sense of (2.11) and (2.12) over fields of characteristic larger than 7 .

2.2. $(G, H)$-sections. We will now follow part of a beautiful procedure investigated by Katsylo in [19] (see also [3]) to prove the rationality of the moduli space of genus 3 curves. It is based on the following notion.

Definition 2.3. Let $G$ be a linear algebraic group acting on an irreducible quasiprojective variety $\mathrm{Y}$. Let $f: H \rightarrow G$ be a group morphism, through which we consider $H$ to act on $\mathrm{Y}$. Let $\mathrm{Z}$ be a closed subvariety of $\mathrm{Y}$. Then $\mathrm{Z}$ is called a $(G, H)$-section of the action of $G$ on $Y$ if

(i) the stabilizer of $\mathrm{Z}$ in $\mathrm{Y}$ is the image of $f$;

(ii) there exists an open subset $Z_{1}$ of $Z$ such that any two points of $Z_{1}$ that are $G$-equivalent in $\mathrm{Y}$ are in fact $H$-equivalent in $\mathrm{Z}$;

(iii) $\mathrm{Y}$ is the closure of $G \cdot \mathrm{Z}$.

Remark 2.4. While the notion of a $(G, H)$-section depends on the morphism from $H$ to $G$, we do not mention this morphism explicitly in what follows.

Proposition $2.5([14, \S 3])$. Suppose that $\mathrm{Z}$ is a $(G, H)$-section of $\mathrm{Y}$. Then the canonical restriction arrow $K(\mathrm{Y})^{G} \rightarrow K(\mathrm{Z})^{H}$ between fields of rational functions is an isomorphism. If we additionally assume that

(i) $\mathrm{Y}$ is an affine normal variety,

(ii) $G$ is a linear algebraic group that does not admit any non-trivial character, and

(iii) any closed orbit of $G$ in $\mathrm{Y}$ intersects $\mathrm{Z}$,

then the canonical restriction arrow $K[\mathrm{Y}]^{G} \rightarrow K[\mathrm{Z}]^{H}$ between rings of regular functions is an isomorphism.

Proof. This proposition is proved in [14, Proposition 4] for the affine case. The generalization of the first part of the result follows from Rosenlicht's theorem [10, Theorem 6.2]; this shows the existence of an invariant affine open in $Z$ whose field 
of $G$-invariant rational functions equals that of Z. The proof in [14, Proposition 4] then still applies.

The situation in which we make use of Proposition 2.5 is the following. We let $\mathrm{Y}$ be the variety whose $K$-points are given by

$$
\mathrm{Y}(K)=\left\{F \in \operatorname{Sym}^{4}\left(V^{*}\right): \operatorname{disc}(\boldsymbol{\rho}(F)) \neq 0\right\} .
$$

Note that we may choose any co- or contravariant of order 2 to define $\mathrm{Y}(K)$, but since among these $\boldsymbol{\rho}$ is of smallest degree (namely 4), it is natural to focus on it here. Consequently,

$$
\mathrm{Y}(K)=\left\{F \in \operatorname{Sym}^{4}\left(V^{*}\right): \boldsymbol{I}_{12}(F) \neq 0\right\} .
$$

That is, $\mathrm{Y}$ is the open subvariety of $\operatorname{Sym}^{4}\left(V^{*}\right)$ whose geometric points correspond to those ternary quartics $F$ for which the contravariant $\boldsymbol{\rho}(F)$ does not degenerate. The subvariety $Z$ is given by the locus of quartics on which the contravariant $\rho$ is normalized, so that

$$
\mathrm{Z}(K)=\left\{F \in \operatorname{Sym}^{4}\left(V^{*}\right): \boldsymbol{\rho}(F)=u\left(v_{2}^{2}-v_{1} v_{3}\right) \text { for some } u \in K^{*}\right\} .
$$

We let $G=\operatorname{SL}(V)$. Note that by our restriction to the open affine where $\boldsymbol{I}_{12} \neq 0$ we have that the ring of invariants

$$
K[\mathrm{Y}]^{G}=K\left[\operatorname{Sym}^{4}\left(V^{*}\right)\right]_{I_{12}}^{G}
$$

is the localization of the ring of Dixmier-Ohno invariants with respect to $\boldsymbol{I}_{12}$.

The stabilizer of $\mathrm{Z}$ in $G$ is given by $\left\langle\zeta_{3}\right\rangle \cdot \mathrm{SO}\left(v_{2}^{2}-v_{1} v_{3}\right)$ where the cube root of unity $\zeta_{3}$ acts via its inclusion into $G$ as a multiple of the identity matrix. This stabilizer $\left\langle\zeta_{3}\right\rangle \cdot \mathrm{SO}\left(v_{2}^{2}-v_{1} v_{3}\right)$ is the image of $H=\left\langle\zeta_{3}\right\rangle \cdot \mathrm{SL}(W)$ (where again $\zeta_{3}$ is considered as a multiple of the identity) by the homomorphism

$$
f=h^{*}: H=\left\langle\zeta_{3}\right\rangle \mathrm{SL}(W) \rightarrow \mathrm{SL}(V)=G
$$

given in (2.3). Note that $\mathbf{Z}$ is defined by 5 equations of degree 4 , and that the regular function $\boldsymbol{u}$ on $\mathbf{Z}$ defined by $\boldsymbol{\rho}(F)=\boldsymbol{u}(F)\left(v_{2}^{2}-v_{1} v_{3}\right)$ is invariant under $\mathrm{SO}\left(v_{2}^{2}-v_{1} v_{3}\right)$. It transforms by $\zeta_{3} \cdot \boldsymbol{u}=\zeta_{3} \boldsymbol{u}$ under the action of $\left\langle\zeta_{3}\right\rangle$. Putting all of this together, we obtain the following result.

Lemma 2.6. With definitions and notations as above, $\mathrm{Z}$ is a $(G, H)$-section of $\mathrm{Y}$, and the canonical restriction map $K[\mathrm{Y}]^{G} \rightarrow K[\mathrm{Z}]^{H}$ is an isomorphism. Moreover, we have that $K[\mathrm{Z}]^{\mathrm{SL}(W)}=K[\mathrm{Z}]^{H}[\boldsymbol{u}]$.

Proof. We have already mentioned that hypothesis (i) of Definition 2.3 is satisfied. We can take $Z_{1}=Z$ in (ii) since by covariance any isomorphism between two forms in $\mathbf{Z}$ fixes the form $v_{2}^{2}-v_{1} v_{3}$ up to a scalar. Since $\mathrm{SL}(V)$ acts transitively on non-degenerate quadric curves, we then also have that $Y=G . Z$ so that certainly (iii) holds. To show the second claim, we have to check that the assumptions in Proposition 2.5 hold in our case. Because $Y$ is an open subset of an affine space, we have (i). Moreover $G=\mathrm{SL}(V)$ does not admit any non-trivial character, so that (ii) is also verified. Again (iii) follows from our considerations in Section 2.1; in fact any orbit of $G$ in $Y$ intersects $Z$.

To obtain the final statement of the lemma, it remains to investigate the action of the central factor $\left\langle\zeta_{3}\right\rangle$ of $H$ on the ring $K[\mathrm{Z}]^{\mathrm{SL}(W)}$. We can decompose

$$
K[\mathrm{Z}]^{\mathrm{SL}(W)}=\bigoplus_{i=0}^{2} M_{i}
$$


where $\left\langle\zeta_{3}\right\rangle$ acts on the $i$-th $K$-submodule $M_{i}$ as scalar multiplication by $\zeta_{3}^{i}$. We have $M_{0}=K[\mathrm{Z}]^{H}$, so that our claim follows from the fact that $M_{i}=\boldsymbol{u}^{i} M_{0}$ for $i>0$. This, in turn, is a consequence of $\boldsymbol{u}$ being a unit in $K[\mathrm{Z}]$; indeed, $\boldsymbol{\rho}(F)=$ $\boldsymbol{u}(F) \cdot\left(v_{2}^{2}-v_{1} v_{3}\right)$, and taking the discriminant on both sides, we get $\operatorname{disc} \boldsymbol{\rho}(F)=$ $\boldsymbol{I}_{12}=\boldsymbol{u}(F)^{3} \cdot \operatorname{disc}\left(v_{2}^{2}-v_{1} v_{3}\right)($ see also $(2.28))$.

Now let $Y^{\prime}=\operatorname{Sym}^{8}\left(W^{*}\right) \oplus \operatorname{Sym}^{4}\left(W^{*}\right) \oplus \operatorname{Sym}^{0}\left(W^{*}\right)$. In Section 2.1 we have seen that there is an isomorphism of $\operatorname{SL}(W)$-representations $\ell^{*}: Y \rightarrow Y^{\prime}$. Let $Z^{\prime}$ be the image of $Z$ under the isomorphism $\ell^{*}$. Then $\ell^{*}$ induces an isomorphism $K[\mathrm{Z}]^{\mathrm{SL}(W)} \rightarrow K\left[\mathrm{Z}^{\prime}\right]^{\mathrm{SL}(W)}$.

Let $\boldsymbol{j}$ be the restriction to $\mathrm{Z}^{\prime}$ of a degree $d$ joint invariant of $\mathrm{SL}(W) \subset H$ on $\mathrm{Y}^{\prime}$. Then certainly $\left(\boldsymbol{j} \ell^{*}\right)^{3}$ is an $H$-invariant function on $\mathbf{Z}$. Therefore by Proposition 2.5 we can write

$$
\left(\boldsymbol{j} \ell^{*}\right)^{3}=\frac{Q}{\boldsymbol{I}_{12}^{n}}
$$

for some $n \in \mathbb{Z}$ and for some polynomial $\boldsymbol{Q}$ in the Dixmier-Ohno invariants that we may suppose not to be divisible by $\boldsymbol{I}_{12}$. Note that $\boldsymbol{Q}$ is homogeneous because $\boldsymbol{j}$ and $\boldsymbol{I}_{12}$ are.

We see that $\boldsymbol{j} \ell^{*} \in K[Z]^{\mathrm{SL}(W)}$ acquires a character under the action of $\left\langle\zeta_{3}\right\rangle$. Now recall from the end of the proof of Lemma 2.6 that we have $\boldsymbol{u}^{3}=\boldsymbol{I}_{12} / 4$. Therefore by our hypothesis $\boldsymbol{I}_{12} \nmid \boldsymbol{Q}$ and (2.23) we see that $\boldsymbol{Q}$ is actually in $K[\mathbf{Z}]^{H}$, and we obtain the following.

Proposition 2.7. Let $\boldsymbol{j}$ be a joint invariant of degree $d$ for the action of $\mathrm{SL}(W)$ on $\operatorname{Sym}^{8}\left(W^{*}\right) \oplus \operatorname{Sym}^{4}\left(W^{*}\right) \oplus \operatorname{Sym}^{0}\left(W^{*}\right)$. Then the composition $\boldsymbol{j} \ell^{*}$, considered as a regular function on $\mathrm{Z} \subset \mathrm{Y}$, admits an expression of homogeneous degree $d$ of the form

$$
\boldsymbol{j} \ell^{*}=\frac{\boldsymbol{P}}{\boldsymbol{u}^{n}}
$$

where $n \geq 0$ and where $\boldsymbol{P}$ is a polynomial in the Dixmier-Ohno invariants of homogeneous degree $d+4 n$. Moreover, $\left(\boldsymbol{j} \ell^{*}\right)^{3}$ extends to $\mathrm{Y}$, where it is described by an expression of homogeneous degree $3 d$ of the form

$$
\left(\boldsymbol{j} \ell^{*}\right)^{3}=\frac{\boldsymbol{P}^{3}}{\boldsymbol{I}_{12}^{n}} .
$$

We need to analyze the pole of $\boldsymbol{j}$ at the zero locus of $\boldsymbol{u}$. In order to do this, we will construct an "association" $\mathrm{Y} \rightarrow \mathrm{Z}$ that, given a plane quartic $F$ corresponding to a point of $Y$, returns an isomorphic quartic in $Z$. This association, while not a morphism of varieties, is still defined by algebraic means in terms of universal forms. Moreover, it does descend to give a well-defined morphism of quotient spaces. Its construction is the objective of the next section, which will also show, among other things, that for the $\mathrm{SL}(W)$-invariant function $\boldsymbol{b}_{0}$ that is homogeneous of degree 1 we have that $\boldsymbol{I}_{9}=5 \boldsymbol{u}^{2} \cdot\left(\boldsymbol{b}_{0} \ell^{*}\right)$ (cf. infra, Lemma 2.12).

2.3. Normalizing quadrics. In order to prove our results and ultimately to perform our computations, we need to transform a generic contravariant quadric into a multiple of $v_{2}^{2}-v_{1} v_{3}$. To do this, we forget about contravariants for a moment and start out with the universal ternary quadric form

$$
\boldsymbol{Q}=a x_{2}^{2}+b x_{2} x_{1}+c x_{2} x_{3}+d x_{1}^{2}+e x_{1} x_{3}+f x_{3}^{2}
$$


over $K$. We want to find a universal linear transformation that transforms $\boldsymbol{Q}$ to a form $\widetilde{\boldsymbol{Q}}=u\left(x_{2}^{2}-x_{1} x_{3}\right)$ with $u \in K[a, b, c, d, e, f]$. Concretely, this will be a linear transformation over a finite extension of $K(a, b, c, d, e, f)$ that sends $\boldsymbol{Q}$ to a non-zero multiple of $x_{2}^{2}-x_{1} x_{3}$.

We will construct this change of variables in such a way that:

(i) we only take one square root;

(ii) the coefficients of the transformations are homogeneous of degree zero in the coefficients of $\boldsymbol{Q}$;

(iii) $\boldsymbol{u}$ is a monomial of small degree (in fact we will have $\boldsymbol{u}=a$ ).

As our transformations will involve a square root, we need to define the degree of an expression involving such roots in order to make sense of (ii) above.

Definition 2.8. Consider a ring $R=K\left[a_{1}, \ldots, a_{n}, r\right]$, where $a_{i}$ are algebraically independent variables and where $r$ is a root of an irreducible polynomial $x^{m}+$ $c_{1} x^{m-1}+\ldots+c_{m}$ such the $c_{i}$ are homogeneous elements of $K\left(a_{1}, \ldots, a_{n}\right)$ whose degree is linear in $i$. We define the homogeneous degrees of the $a_{i}$ to be 1 , and that of $r$ to be $\operatorname{deg}\left(c_{m}\right) / m$. The homogeneous degree of an element $r$ of $R$, is defined to be the common homogeneous degree of the monomials in a representation of $r$, if such a common degree exists.

Note that this definition is independent of the choice of representation of $r$ because of the properties of the minimal polynomial of $r$. We obtain a decomposition $R=\oplus_{d} R_{d}$ by homogeneous degree. We extend this grading to the fraction field of $R$ in the usual way. In particular, a quotient of the form $P / Q$ of homogeneous polynomials $P$ and $Q$ of $\operatorname{degree} \operatorname{deg}(P)$, respectively $\operatorname{deg}(Q)$, is said to have homogeneous degree $\operatorname{deg}(P)-\operatorname{deg}(Q)$.

Let $\delta=a d-b^{2} / 4, \eta=a e-b c / 2$, and

$$
\boldsymbol{\Delta}=a d f-a e^{2} / 4-b^{2} f / 4+b c e / 4-c^{2} d / 4
$$

so that $\boldsymbol{\Delta}$ is the discriminant of $\boldsymbol{Q}$. To obtain our normalization, we proceed as follows:

(i) eliminate the monomials $x_{2} x_{1}$ and $x_{2} x_{3}$ of $\boldsymbol{Q}$ by applying the transformation

$$
x_{2} \leftarrow x_{2}-\frac{b}{2 a} x_{1}-\frac{c}{2 a} x_{3} .
$$

We call the result $Q_{1}$.

(ii) eliminate the monomial $x_{1} x_{3}$ in $Q_{1}$ by applying

$$
x_{1} \leftarrow x_{1}-\frac{\eta}{2 \delta} x_{3} .
$$

We call the result $Q_{2}$.

(iii) Let $r$ be such that $r^{2}+a \boldsymbol{\Delta}=0$ (so that $r$ has homogeneous degree 2). Transform $Q_{2}$ into $Q_{3}=a x_{2}^{2}+(\delta / a) x_{1} x_{3}$ by applying

$$
x_{1} \leftarrow \frac{x_{1}+x_{3}}{2}, \quad x_{3} \leftarrow \frac{\delta}{2 r}\left(x_{1}-x_{3}\right) .
$$

(iv) Transform $Q_{3}$ into normalized form by applying

$$
x_{1} \leftarrow-\frac{\Delta}{a^{2}} x_{1} .
$$


Composing all these maps, we obtain that $T_{0} \cdot \boldsymbol{Q}$ is a multiple of $x_{2}^{2}-x_{1} x_{3}$, where

$$
T_{0}=\left(\begin{array}{ccc}
-\frac{\delta}{a^{2}} & 0 & -\frac{2 r+\eta}{2 a^{2}} \\
\frac{b}{2 a} & 1 & \frac{c}{2 a} \\
1 & 0 & -\frac{r-\eta}{\delta}
\end{array}\right)
$$

When applying this to a contravariant, as we will indeed do later, we instead have to transform by applying $T_{0}^{-1}$ on the right.

For later use, we also define the integral matrix

$$
T_{\text {int }}=a^{3} \delta^{2} T_{0}
$$

One computes

$$
\begin{aligned}
\operatorname{det}\left(T_{\mathrm{int}}\right)^{2} & =\left(a^{9} \delta^{6}\right)^{2} \operatorname{det}\left(T_{0}\right)^{2} \\
& =\left(a^{9} \delta^{6}\right)^{2} \cdot 2^{2} r^{2} / a^{4} \\
& =\quad-2^{2} a^{15} \delta^{12} \boldsymbol{\Delta}
\end{aligned}
$$

Note how the matrices constructed in this section depend on the choice of a square root. Because of this, they do not immediately give rise to morphisms of varieties. However, we will see later ( $c f$. infra, Theorem 2.16) that they can be used to construct functions that are honest morphisms, and that even admit expressions in the Dixmier-Ohno invariants.

Remark 2.9. It is in fact possible to get integral matrices $T$ of smaller homogeneous weight. The best method known to us multiplies $T_{0}$ by a matrix over a larger extension whose determinant is homogeneous of degree 6 in the coefficients. We do not need this more effective integral version in what follows.

2.4. Extension from $Z$ to $Y$. In this section we describe how Proposition 2.7 can be extended from $Z$ to all of $Y$. Let $T$ be a universal transformation (as defined in the previous section) that sends $\rho$ to a non-zero multiple of $v_{2}^{2}-v_{1} v_{3}$. With the notation of Proposition 2.7, we can then consider the functions $j \ell^{*} T$ and $\boldsymbol{b}_{0} \ell^{*} T$ on Y.

Proposition 2.10. Let $\boldsymbol{j}$ be a joint invariant of degree $d$. Then the functions $\boldsymbol{j} \ell^{*} T$ and $\boldsymbol{b}_{0} \ell^{*} T$ on $\mathrm{Y}$ satisfy the following properties:

(i) the quotient $\boldsymbol{j} \ell^{*} T /\left(\boldsymbol{b}_{0} \ell^{*} T\right)^{d}$ is $\mathrm{GL}(V)$-invariant and does not depend on the choice of $T$;

(ii) for $T=T_{0}$ the functions $\boldsymbol{j} \ell^{*} T$ and $\boldsymbol{b}_{0} \ell^{*} T$ are in $K\left(\operatorname{Sym}^{4}\left(V^{*}\right)\right)$;

(iii) for $T=T_{\mathrm{int}}$ the functions $\boldsymbol{j} \ell^{*} T$ and $\boldsymbol{b}_{0} \ell^{*} T$ are in $K\left[\operatorname{Sym}^{4}\left(V^{*}\right)\right]$.

Proof. (i): Any two possible $T$ differ by an element of $\operatorname{GO}\left(v_{2}^{2}-v_{1} v_{3}\right)$, the image of $\mathrm{GL}(W)$ under $h^{*}$. The result follows because $\boldsymbol{j}$ and $\boldsymbol{b}_{0}$ are invariant under $\mathrm{SL}(W)$ and $\boldsymbol{j} / \boldsymbol{b}_{0}^{d}$ is homogeneous of weight 0 .

(ii): The matrix $T_{0}$ is a priori defined over a quadratic extension of $K\left[\operatorname{Sym}^{4}\left(V^{*}\right)\right]$, as are therefore the functions $\boldsymbol{j} \ell^{*} T$ and $\boldsymbol{b}_{0} \ell^{*} T$. By Galois theory it suffices to show that applying its quadratic conjugate $T_{0}^{\sigma}$ to the universal ternary quartic yields the same result. One calculates first of all that $\operatorname{det}\left(T_{0}^{\sigma}\right)=-\operatorname{det}\left(T_{0}\right)$. This means that $T_{0}$ and $T_{0}^{\sigma}$ differ by the product of an element of $\mathrm{SO}\left(v_{2}^{2}-v_{1} v_{3}\right)$ and the scalar matrix 
-1 . Now -1 is central and its action on quartic forms is trivial. The conclusion therefore follows from the fact that $\boldsymbol{j}$ and $\boldsymbol{b}_{0}$ are invariant under $\operatorname{SL}(W)$.

(iii): This follows by combining the argument in the proof of (ii) with the fact that the polynomial ring $K\left[\operatorname{Sym}^{4}\left(V^{*}\right)\right]$ is integrally closed in its field of fractions $K\left[\operatorname{Sym}^{4}\left(V^{*}\right)\right]$.

Remark 2.11. Note that in general $\boldsymbol{j} \ell^{*} T$ and $\boldsymbol{b}_{0} \ell^{*} T$ contain superfluous factors and are far from being $\mathrm{SL}(V)$-invariant themselves.

2.5. A fundamental lemma. Let $\boldsymbol{F}$ be the universal ternary quartic, and let $\boldsymbol{\rho}$ be the contravariant defined in (1.23). As in the previous section, let $T$ be an algebraic transformation matrix that sends $\boldsymbol{\rho}$ to the multiple $\boldsymbol{u} \cdot\left(v_{2}^{2}-v_{1} v_{3}\right)$, or more precisely such that we have

$$
\boldsymbol{\rho}(\underline{a}, \underline{v} \cdot T)=\boldsymbol{u} \cdot\left(v_{2}^{2}-v_{1} v_{3}\right) .
$$

We can then define $\widetilde{\boldsymbol{F}}=\boldsymbol{F} . T=\boldsymbol{F}(\underline{a}, \underline{x} . T)=\boldsymbol{F}(T \cdot \underline{a}, \underline{x})$. Since the covariant $\boldsymbol{\rho}$ is homogeneous of weight equals $(4 \cdot 4+2) / 3=6$, Eq. (1.14) shows that

$$
\widetilde{\boldsymbol{\rho}}:=\boldsymbol{\rho}(\widetilde{\boldsymbol{F}})=\boldsymbol{\rho}(T \cdot \underline{a}, \underline{v})=\operatorname{det}(T)^{6} \cdot \boldsymbol{\rho}(\underline{a}, \underline{v} \cdot T)=\operatorname{det}(T)^{6} \cdot \boldsymbol{u} \cdot\left(v_{2}^{2}-v_{1} v_{3}\right) .
$$

Using the definitions of the operators in Section 1.2, we see that

$$
\begin{array}{r}
\widetilde{\boldsymbol{T}}:=\boldsymbol{T}(\widetilde{\boldsymbol{F}}, v)=D(\widetilde{\boldsymbol{\rho}}, \widetilde{\boldsymbol{F}}) / 12=\operatorname{det}(T)^{6} \cdot D(\boldsymbol{\rho}(\underline{a}, \underline{v} \cdot T), \widetilde{\boldsymbol{F}}) / 12, \\
\widetilde{\boldsymbol{I}}_{9}:=\boldsymbol{I}_{9}(\widetilde{\boldsymbol{F}})=J_{11}(\widetilde{\boldsymbol{T}}, \widetilde{\boldsymbol{\rho}})=\operatorname{det}(T)^{6} \cdot J_{11}(\widetilde{\boldsymbol{T}}, \boldsymbol{\rho}(\underline{a}, \underline{v} \cdot T)) .
\end{array}
$$

By using the particular shape of $\boldsymbol{\rho}(\underline{a}, \underline{v} . T)$, we calculate that in terms of the coefficients $\widetilde{a}$ of $\widetilde{\boldsymbol{F}}$ we have that

$$
\widetilde{\boldsymbol{I}}_{9}=\operatorname{det}(T)^{12} \cdot \boldsymbol{u}^{2} \cdot\left(\frac{\widetilde{a}_{202}}{6}-\frac{\widetilde{a}_{121}}{6}+\widetilde{a}_{040}\right) .
$$

Since $\boldsymbol{I}_{9}$ is of weight $9 \cdot 4 / 3=12,(1.14)$ also gives that

$$
\boldsymbol{I}_{9}=\operatorname{det}(T)^{-12} \cdot \widetilde{\boldsymbol{I}}_{9}=\boldsymbol{u}^{2} \cdot\left(\frac{\widetilde{a}_{202}}{6}-\frac{\widetilde{a}_{121}}{6}+\widetilde{a}_{040}\right) .
$$

On the other hand, using the equivariant isomorphism $\ell^{*}$ from (2.10) we see that in terms of the coefficients $\underline{\widetilde{a}}$ of $\widetilde{F}$, the constant binary form $\boldsymbol{b}_{0} \ell^{*} T$ is given by

$$
\boldsymbol{b}_{0} \ell^{*} T=\frac{\widetilde{a}_{202}}{30}-\frac{\widetilde{a}_{121}}{30}+\frac{\widetilde{a}_{040}}{5} .
$$

Thus we obtain the algebraic equality

$$
\boldsymbol{I}_{9}=5 \cdot \boldsymbol{u}^{2} \cdot\left(\boldsymbol{b}_{0} \ell^{*} T\right) .
$$

Finally, from Section 1.2 we know that the discriminant of $\boldsymbol{\rho}$ equals $\boldsymbol{I}_{12}$ whereas the discriminant of $\boldsymbol{\rho}(\underline{a}, \underline{v} \cdot T)$ equals $-\boldsymbol{u}^{3} / 4$ by $(2.36)$. Since $\boldsymbol{\rho}(\underline{a}, \underline{v} \cdot T)=\boldsymbol{\rho}\left(\underline{a}, T^{-1} \cdot \underline{v}\right)$, we see that the matrix $[\boldsymbol{\rho}(\underline{a}, \underline{v} . T)]$ that represents the symmetric bilinear form corresponding to the quadric contravariant $\rho(\underline{a}, \underline{v} . T)$ can be obtained from the matrix $[\boldsymbol{\rho}]$ that represents $\rho$ as $T^{-1}[\boldsymbol{\rho}]\left(T^{-1}\right)^{t}$. Taking the discriminant comes down to taking the determinant of these forms, and therefore

$$
\begin{aligned}
-\boldsymbol{u}^{3} / 4 & =\operatorname{disc}(\boldsymbol{\rho}(\underline{a}, \underline{v} \cdot T))=\operatorname{det}([\boldsymbol{\rho}(\underline{a}, \underline{v} \cdot T)])=\operatorname{det}\left(T^{-1} \cdot[\boldsymbol{\rho}(\underline{a}, \underline{v})] \cdot\left(T^{-1}\right)^{t}\right) \\
& =\operatorname{det}\left(T^{-1}\right)^{2} \operatorname{det}([\boldsymbol{\rho}(\underline{a}, \underline{v})])=\operatorname{det}\left(T^{-1}\right)^{2} \operatorname{disc}(\boldsymbol{\rho}(\underline{a}, \underline{v})) \\
& =\boldsymbol{I}_{12} / \operatorname{det}(T)^{2} .
\end{aligned}
$$


Since on $\mathrm{Z}$ we can moreover take $T$ to be the identity, (2.42) and (2.43) translate into the following result, which will be fundamental to our study of the integrality of the functions $\boldsymbol{j} \ell^{*} T /\left(\boldsymbol{b}_{0} \ell^{*} T\right)^{d}$.

Lemma 2.12. We have the equalities

$$
\boldsymbol{I}_{9}=5 \cdot \boldsymbol{u}^{2} \cdot\left(\boldsymbol{b}_{0} \ell^{*}\right)
$$

of functions on $\mathrm{Z}$ and

$$
\left(\frac{\boldsymbol{I}_{9}}{\boldsymbol{b}_{0} \ell^{*} T}\right)^{3}=2000 \cdot\left(\frac{\boldsymbol{I}_{12}}{\operatorname{det}(T)^{2}}\right)^{2}
$$

of functions on $\mathrm{Y}$.

2.6. Denominator bounds. It turns out that the results of the previous sections give us a way to bound the denominators of the functions $\boldsymbol{j} \ell^{*}$ on $\mathrm{Z}$ from Proposition 2.7. Moreover, we can describe the functions $\boldsymbol{j} \ell^{*} T /\left(\boldsymbol{b}_{0} \ell^{*} T\right)^{d}$ on $\mathrm{Y}$ as well. As a first step, we prove two lemmata.

Lemma 2.13. We have the following.

(i) Let $S=P / Q$ be a simplified fraction in $K\left(\operatorname{Sym}^{4}\left(V^{*}\right)\right)$. If $S$ is invariant under $\mathrm{SL}(V)$, then so are the elements $P$ and $Q$ of $K\left[\operatorname{Sym}^{4}\left(V^{*}\right)\right]$.

(ii) Let $P \in K\left[\operatorname{Sym}^{4}\left(V^{*}\right)\right]$. If $P$ is invariant under $\mathrm{SL}(V)$, then so are all its irreducible factors in the ring $K\left[\operatorname{Sym}^{4}\left(V^{*}\right)\right]$.

(iii) The ring $K\left[\operatorname{Sym}^{4}\left(V^{*}\right)\right]^{\mathrm{SL}(V)}$ is a unique factorization domain.

Proof. (i) (See also [10, Exercise 6.10].) Because the fraction $S$ is simplified, the divisors defined by $P$ and $Q$ are invariant under $\mathrm{SL}(V)$. Therefore the action of $\mathrm{SL}(V)$ is described by multiplication with a character. But the group $\mathrm{SL}(V)$ has no non-trivial character.

(ii) Consider the locus $P=0$ in the affine space over $\operatorname{Sym}^{4}(V)$. This locus consists of a finite number of irreducible components. The corresponding stabilizers are subgroups of SL $(V)$ of finite index. These subgroups give rise to the full tangent space at the origin. By the classical correspondence between Lie subgroups and Lie subalgebras, they therefore correspond to a connected Lie subgroup of $\operatorname{SL}(V)$ of the same dimension as the latter group. But since $\operatorname{SL}(V)$ is irreducible, it is certainly connected, so we see that they all coincide with $\operatorname{SL}(V)$. This shows that the irreducible components of the locus $P=0$ are invariant under $\mathrm{SL}(V)$.

The irreducible factors of $P$ generate the radical ideals of the corresponding irreducible components, and are characterized up to a non-zero scalar by this property. Since the action of $\mathrm{SL}(V)$ preserves degree, we see that in fact these factors transform by a character under the action of $\mathrm{SL}(V)$. We can therefore again conclude by using the fact that $\mathrm{SL}(V)$ has no non-trivial character.

Part (iii) is a consequence of part (ii).

Remark 2.14. The fact that $K\left[\operatorname{Sym}^{4}\left(V^{*}\right)\right]^{\mathrm{SL}(V)}$ is a unique factorization domain was known to Hilbert [18, II.3]. General results in this direction (proved by different methods than those used in Lemma 2.13) were also obtained by Popov [31] and Hashimoto [17].

Lemma 2.15. The element $\boldsymbol{I}_{12}$ of $K\left[\operatorname{Sym}^{4}\left(V^{*}\right)\right]$ is irreducible. 
Proof. By Lemma 2.13-(i), it suffices to show irreducibility of $\boldsymbol{I}_{12}$ in the ring of Dixmier-Ohno invariants $K\left[\operatorname{Sym}^{4}\left(V^{*}\right)\right]^{\mathrm{SL}(V)}$, and one can indeed verify by linear algebra and interpolation ( $c f$. Section 2.8) that no expression of $\boldsymbol{I}_{12}$ in terms of invariants of lower homogeneous degree exists.

Now we can state the following stronger version of Proposition 2.7.

Theorem 2.16. Let $\boldsymbol{j}$ be a joint invariant of total degree $d$. With the notation of Section 2.2, on $\mathbf{Z}$ we have

$$
\boldsymbol{j} \ell^{*}=\frac{\boldsymbol{P}}{\boldsymbol{u}^{2 d}}
$$

where $\boldsymbol{P}$ is a polynomial in the Dixmier-Ohno invariants that is homogeneous of degree $9 d$. Moreover, we have an equality

$$
\frac{\boldsymbol{j} \ell^{*} T}{\left(\boldsymbol{b}_{0} \ell^{*} T\right)^{d}}=\frac{\boldsymbol{P}}{\boldsymbol{I}_{9}^{d}}
$$

of functions on $\mathrm{Y}$.

Proof. Consider $\left(\boldsymbol{j} \ell^{*}\right)^{3}$ as a function on $\mathrm{Y}$. Then by Proposition 2.7 we have $\left(\boldsymbol{j} \ell^{*}\right)^{3}=\boldsymbol{P}^{3} / \boldsymbol{I}_{12}^{n}$ for some $n$ and hence it is invariant by $\mathrm{SL}(V)$ Now the expression $\left(\boldsymbol{j} \ell^{*}\right)^{3}$ is of homogeneous degree $3 d$ in the coefficients, it is homogeneous of weight $4 d$. Therefore by Lemma 2.12 we have

$$
\left(\boldsymbol{j} \ell^{*} T\right)^{3}=\operatorname{det}(T)^{4 d}(\boldsymbol{j} \ell)^{3}=\operatorname{det}(T)^{4 d} \frac{\boldsymbol{P}}{\boldsymbol{I}_{12}^{n}}=\frac{\boldsymbol{P}}{\left(\left(\boldsymbol{I}_{9} / \boldsymbol{b}_{0} \ell^{*} T\right)^{3 d} \boldsymbol{I}_{12}^{n-2 d}\right)} .
$$

We already know $\boldsymbol{j} \ell^{*} T /\left(\boldsymbol{b}_{0} \ell^{*} T\right)^{d}$ to be rational from Proposition 2.10 - (i). Now we take $T=T_{\text {int }}$, so that both $\boldsymbol{j} \ell^{*} T_{\text {int }}$ and $\boldsymbol{b}_{0} \ell^{*} T_{\text {int }}$ are polynomials. By Lemma 2.13, we can then rewrite (2.48) as a polynomial equality in the unique factorization domain $K\left[\operatorname{Sym}^{4}\left(V^{*}\right)\right]$, where it becomes

$$
\boldsymbol{I}_{9}^{3 d} \cdot\left(\boldsymbol{j} \ell^{*} T_{\mathrm{int}}\right)^{3} \cdot \boldsymbol{I}_{12}^{n-2 d}=\boldsymbol{P} \cdot\left(\boldsymbol{b}_{0} \ell^{*} T_{\mathrm{int}}\right)^{3 d} .
$$

By Lemma 2.15 , if we show that $\boldsymbol{I}_{12}$ does not actually divide the polynomial $\boldsymbol{b}_{0} \ell^{*} T$, then we get that $n \leq 2 d$. One verifies this by finding a single plane quartic $F$ for which $\boldsymbol{b}_{0} \ell^{*} T_{\text {int }}(F) \neq 0$ while $\boldsymbol{I}_{12}(F)=0$, which can be done by using a computer algebra system. This proves that one has

$$
\frac{\boldsymbol{j} \ell^{*} T}{\left(\boldsymbol{b}_{0} \ell^{*} T\right)^{d}}=\frac{\boldsymbol{P} \cdot \boldsymbol{I}_{12}^{s}}{\boldsymbol{I}_{9}^{d}}
$$

with $s$ non-negative. This yields the second statement of the theorem on $\mathrm{Y}$. Transforming back, one obtains the first statement on Z.

Remark 2.17. As we see, the functions $\boldsymbol{j} \ell^{*}$ themselves do not admit a rational expression in the Dixmier-Ohno invariants in $Z$ and therefore do not extend to an invariant function on $Y$. The group-theoretic reason for this is that under the action of $\mathrm{SL}(V)$ the normalization of $\boldsymbol{\rho}$ is only determined up to a factor $\zeta_{3}$, making the function $\boldsymbol{u}$ ill-defined on $\mathrm{Y}$; only $\boldsymbol{u}^{3}$ extends to a function on $\mathrm{Y}$, and with it $\left(\boldsymbol{j} \ell^{*}\right)^{3}$. On Z, where $\boldsymbol{u}$ can be immediately recovered from the quartic by taking its covariant, this problem does not occur by construction.

Remark 2.18. The functions $\boldsymbol{j} \ell^{*} T /\left(\boldsymbol{b}_{0} \ell^{*} T\right)^{d}$ also extend to $\mathrm{Y}$ because the characters of the numerator and the denominator under the action of $\left\langle\zeta_{3}\right\rangle$ cancel. Note that for our reconstruction purposes we can use either of these formulas; the mutual 
quotients of the joint invariants suffice because geometrically we are only interested in quartics up to a scalar.

2.7. A projective approach. It is possible to obtain the second result of Theorem 2.16 without intervention of the group $\left\langle\zeta_{3}\right\rangle$, at least if one is willing to work with projective spaces.

In order to achieve this, we use the inclusion $\mathbb{P} Z \subset \mathbb{P Y}$ instead, which is a $(G, H)$ section for the group morphism $h^{*}: \operatorname{PSL}(W) \rightarrow \operatorname{PSL}(V)$ with image $\mathrm{SO}(V)$. Using the equivariant isomorphism (2.10) and the first part of Proposition 2.5, we see that $\left(\boldsymbol{j} \ell^{*}\right) /\left(\boldsymbol{b}_{0} \ell^{*}\right)^{d}=\boldsymbol{P} / \boldsymbol{Q} \in K(\mathbb{P} Y)^{G}$, where $\boldsymbol{P}$ and $\boldsymbol{Q}$ are polynomials in the Dixmier-Ohno invariants that are homogeneous of the same degree.

We now shrink $\mathbb{P} Y$ to $\mathbb{P}^{\prime} Y=\mathbb{P} \operatorname{Sym}^{4}\left(V^{*}\right) \backslash\left(\left\{\boldsymbol{I}_{12}=0\right\} \cup\left\{\boldsymbol{I}_{9}=0\right\}\right)$ and define $\mathbb{P}^{\prime} Z$ correspondingly. By Lemma 2.12 the function $\boldsymbol{b}_{0} \ell^{*}$ is non-zero on the representatives of elements of $\mathbb{P}^{\prime} Z$. Since $\mathbb{P}^{\prime} Y$ is normal and affine we can use the second part of Proposition 2.5 to prove the following lemma.

Lemma 2.19. There exist $s, t \in \mathbb{N}$ and a polynomial $\boldsymbol{P}$ in the Dixmier-Ohno that is homogeneous of degree $12 s+9 t$ such that

$$
\frac{\boldsymbol{j} \ell^{*}}{\left(\boldsymbol{b}_{0} \ell^{*}\right)^{d}}=\frac{\boldsymbol{P}}{\boldsymbol{I}_{12}^{s} \cdot \boldsymbol{I}_{9}^{t}}
$$

on $\mathbb{P}^{\prime} \mathrm{Z}$.

If we use $T=T_{\text {int }}$, we have proved in Proposition 2.10 that $j \ell^{*} T$ and $\boldsymbol{b}_{0} \ell^{*} T$ belong to $K\left[\operatorname{Sym}^{4}\left(V^{*}\right)\right]$. Moreover

$$
\frac{\boldsymbol{j} \ell^{*} T}{\left(\boldsymbol{b}_{0} \ell^{*} T\right)^{d}}=\frac{\boldsymbol{P} \cdot T}{\left(\boldsymbol{I}_{12} \cdot T\right)^{s} \cdot\left(\boldsymbol{I}_{9} \cdot T\right)^{t}}=\frac{\boldsymbol{P}}{\boldsymbol{I}_{12}^{s} \cdot \boldsymbol{I}_{9}^{t}}
$$

by invariance of the denominator and numerator under $\mathrm{SL}(V)$ and the fact that they have the same degree. Hence, in the unique factorization domain $\mathbb{C}\left[\operatorname{Sym}^{4}\left(V^{*}\right)\right]$ we have the equality of polynomials

$$
\left(\boldsymbol{j} \ell^{*} T_{\text {int }}\right) \cdot\left(\boldsymbol{I}_{12}^{s} \cdot \boldsymbol{I}_{9}^{t}\right)=\boldsymbol{P} \cdot\left(\boldsymbol{b}_{0} \ell^{*} T_{\text {int }}\right)^{d} .
$$

As before, one verifies that $\boldsymbol{b}_{0} \ell^{*} T_{\mathrm{int}}$ is not divisible by $\boldsymbol{I}_{12}$ by finding a single plane quartic $F$ for which $\boldsymbol{b}_{0} \ell^{*} T_{\text {int }}(F)$ is non-zero while $\boldsymbol{I}_{12}(F)=0$. Hence $\boldsymbol{I}_{12}^{s}$ divides $\boldsymbol{P}$. If we denote $\boldsymbol{P}_{1}=\boldsymbol{P} / \boldsymbol{I}_{12}^{s}$, since $\boldsymbol{P}$ and $\boldsymbol{I}_{12}^{s}$ are invariants under the action of $\mathrm{SL}(V), \boldsymbol{P}_{1}$ is as well and is therefore a polynomial in the Dixmier-Ohno invariants. We can also assume, after a possible division, that $\boldsymbol{P}_{1}$ is coprime to $\boldsymbol{I}_{9}$ and we can write $\left(\boldsymbol{j} \ell^{*}\right) /\left(\boldsymbol{b}_{0} \ell^{*}\right)^{d}=\boldsymbol{P}_{1} / \boldsymbol{I}_{9}^{t_{1}}$ with $t_{1} \in \mathbb{N}$.

To conclude, we only have to prove that $t_{1}=d$. Again we have

$$
\frac{\boldsymbol{j} \ell^{*} T_{\mathrm{int}}}{\left(\boldsymbol{b}_{0} \ell^{*} T_{\mathrm{int}}\right)^{d}}=\frac{\boldsymbol{P}_{1}}{\boldsymbol{I}_{9}^{t_{1}}}
$$

which gives

$$
\boldsymbol{j} \ell^{*} T_{\mathrm{int}}=\frac{\boldsymbol{P}_{1}}{\boldsymbol{I}_{9}^{t_{1}}} \cdot\left(\boldsymbol{b}_{0} \ell^{*} T_{\mathrm{int}}\right)^{d}
$$

From Lemma 2.12 and (2.35) we obtain that, up to a multiplicative constant,

$$
\boldsymbol{b}_{0}^{3 d} \ell^{*} T_{\mathrm{int}}=\boldsymbol{I}_{9}^{3 d} \frac{\operatorname{det}\left(T_{\mathrm{int}}\right)^{4 d}}{\boldsymbol{I}_{12}^{2 d}}=\boldsymbol{I}_{9}^{3 d} \cdot a^{30 d} \delta^{24 d}\left(\frac{\boldsymbol{\Delta}}{\boldsymbol{I}_{12}}\right)^{2 d}=\boldsymbol{I}_{9}^{3 d} \cdot a^{30 d} \delta^{24 d}
$$


since $\boldsymbol{\Delta}=\operatorname{disc}(\boldsymbol{\rho})=\boldsymbol{I}_{12}$. We therefore see that (2.55) translates, up to a constant, into

$$
\boldsymbol{I}_{9}^{3 t_{1}} \cdot\left(\boldsymbol{j} \ell^{*} T_{\mathrm{int}}\right)^{3}=\boldsymbol{I}_{9}^{3 d} \cdot \boldsymbol{P}_{1}^{3} \cdot\left(a^{30 d} \delta^{24 d}\right)
$$

as an equality in $\mathbb{C}\left[\operatorname{Sym}^{4}\left(V^{*}\right)\right]$. To conclude, let us observe that $\boldsymbol{I}_{9}$ is irreducible. Indeed, otherwise it would decompose as a product of invariants by Lemma 2.13(i) and therefore it would be a polynomial in $\boldsymbol{I}_{3}$ and $\boldsymbol{I}_{6}$. This is not possible as $\boldsymbol{I}_{9}, \boldsymbol{I}_{6}, \boldsymbol{I}_{3}$ are algebraically independent. Moreover $a$ and $\delta$ are polynomials of degree less than $\boldsymbol{I}_{9}$, so $\boldsymbol{I}_{9}$ is coprime to $a$ and $\delta$ and by hypothesis to $\boldsymbol{P}_{1}$. Therefore we get $t_{1}=d$, which concludes the proof.

2.8. Evaluation-interpolation. Since the 63 joint invariants given in Table 1 form a minimal basis of the ring of invariants $K\left[\operatorname{Sym}^{8}\left(W^{*}\right) \oplus \operatorname{Sym}^{4}\left(W^{*}\right)\right]^{\mathrm{SL}(W)}$, we proceed to determine the polynomials $\boldsymbol{P}$ in Theorem 2.16 for them. Here general algebraic-geometric considerations do not seem to help us further, and we therefore use a method that has served us well in the past, namely that of evaluationinterpolation [22].

From (2.42) and (2.47), we seek to determine the regular function on $\mathrm{Y}$ defined by $\boldsymbol{j} \ell^{*} T_{0} \times \boldsymbol{I}_{9}^{d} /\left(\boldsymbol{b}_{0} \ell^{*} T_{0}\right)^{d}$ as an homogeneous polynomial $\boldsymbol{P}$ in the Dixmier-Ohno invariants; we may as well restrict to $Z$ because up to the usual scalar the calculated polynomial $\boldsymbol{P}$ is identical. Our strategy is to evaluate $\boldsymbol{P}$ at lots of random quartics $F$ in $\mathrm{Y}$, so that we get equations

$$
\boldsymbol{P}\left(\boldsymbol{I}_{3}(F), \boldsymbol{I}_{6}(F), \ldots, \boldsymbol{I}_{27}(F)\right)=\boldsymbol{j}\left(\ell^{*}(\widetilde{F})\right) \times \frac{\boldsymbol{I}_{9}(F)^{d}}{\boldsymbol{b}_{0}\left(\ell^{*}(\widetilde{F})\right)^{d}} .
$$

Since we know the homogeneous weight $9 d$ of $\boldsymbol{P}$, we can recover it by evaluating it in a known finite number of points. More systematically, our strategy is as follows:

(i) Generate a large family of random quartics $F$;

(ii) Given a quartic $F$ in the family, use either the matrix (2.33) (or (2.34)) from Section 2.3 to determine a quartic $\widetilde{F}$ with normalized covariant $\widetilde{\rho}$;

(iii) Calculate the quotients $\boldsymbol{j}\left(\ell^{*}(\widetilde{F})\right) \boldsymbol{I}_{9}(F)^{d} / \boldsymbol{b}_{0}\left(\ell^{*}(\widetilde{F})\right)^{d}$ as in $(2.58)$ by using our explicit knowledge of $\ell^{*}$ and the joint invariants on $\operatorname{Sym}^{8}(W) \oplus$ $\operatorname{Sym}^{4}(W)$ from Section 1.3;

(iv) Evaluate at $F$ all the monomials of degree $9 d$ in the Dixmier-Ohno invariants;

(v) Determine $\boldsymbol{P}$ by solving the linear equation in its coefficients obtained from the evaluations in (iii) and (iv).

The interpolation in the final step amounts to a search for a polynomial that is homogeneous of degree $9 d$ in the Dixmier-Ohno invariants such that its values coincide with those of $\boldsymbol{j}\left(\ell^{*}(\widetilde{F})\right) \boldsymbol{I}_{9}^{d}(F) / \boldsymbol{b}_{0}\left(\ell^{*}(\widetilde{F})\right)^{d}$. The most expensive computation in this approach is the matrix inversion, the complexity of which depends of the dimension of the matrix, i.e. the number of monomials of degree $9 d$ in the Dixmier-Ohno invariants. This number is equal to the coefficient of $x^{9 d}$ in the series expansion of $\prod\left(1-x^{\operatorname{deg}(\boldsymbol{I})}\right)^{-1}$ where the $\boldsymbol{I}$ are the Dixmier-Ohno invariants. In the largest computations that we have done, we had $d=10$, which gives a matrix of dimension 27398. The size of the required family of quartics should be larger than this dimension; taking one hundred more is enough in practice.

An additional complication is that the Dixmier-Ohno invariants satisfy certain relations. This means that in order for $\boldsymbol{P}$ to be unique we have to restrict to a 
subset of the monomials considered above, the size of which is given by a coefficient of the Hilbert series of the ring of Dixmier-Ohno invariants, which was determined in [35]. While this coefficient can indeed be computed, determining a correct subset amounts to calculating a Gröbner basis of the ideal of relations. This still seems to be beyond reach. We therefore run into the additional complication that the polynomial $\boldsymbol{P}$ that we obtain is not unique. For example, because of the relations between the Dixmier-Ohno invariants the interpolation matrix already has a kernel of dimension 14861 for $d=10$.

Directly inverting the matrix over a field of characteristic 0 turns out to be a rather suboptimal approach, since one then quickly runs out of memory. Moreover, the heights of the corresponding polynomial expression also increases rapidly, with a numerator and denominator of about 270 decimal digits when $d=10$. To ease our computations, we have therefore used another classical trick, namely the determination of the coefficients of $\boldsymbol{P}$ modulo a sufficiently large amount of 9-digit primes. Using the Chinese remainder theorem then allowed us to recover a hypothetical solution over $\mathbb{Q}$.

Once a conjectural expression for $\boldsymbol{P}$ has been obtained, the result can be verified to be correct a posteriori, that is, by checking that it gives the correct answer on a sufficiently large set of ternary quartics. More precisely, our procedure to verify the conjectural expression of a joint invariant $\boldsymbol{j}$ of degree $d$ is as follows.

(i) Calculate the monomials $B$ of weight $9 d$ in the Dixmier-Ohno invariants;

(ii) Generate a large family $S$ of random quartics $F$;

(iii) Verify that the interpolated forms over $\mathbb{Q}$ are correct for the quartics in $S$, in the sense that (2.58) holds for all $F$ in $S$; if not, terminate and indicate that the interpolation is incorrect;

(iv) Form the matrix $M$ obtained by evaluating the monomials in $B$ at the quartics in $S$;

(v) If the rank of $M$ equals the $9 d$-th coefficient of the Hilbert series of the ring of Dixmier-Ohno invariants, terminate and return that the interpolation is correct; otherwise add more quartics to $S$ and start again at step (ii).

An especially pleasant feature of this verification algorithm is that it again suffices to determine the rank of the matrix $M$ over a field of small characteristic; this greatly speeds up the calculations.

\begin{tabular}{c|c|c|r|r} 
Degree $d$ & Dimension & $\#$ digits & Timings & Size (bytes) \\
\hline \hline 2 & 19 & 28 & $0 \mathrm{sec}$ & $\simeq 50 \mathrm{~b}$ \\
3 & 67 & 46 & $1 \mathrm{sec}$ & $\simeq 1 \mathrm{~kb}$ \\
4 & 206 & 64 & $5 \mathrm{sec}$ & $\simeq 2,5 \mathrm{~kb}$ \\
5 & 557 & 91 & $20 \mathrm{sec}$ & $\simeq 10 \mathrm{~kb}$ \\
6 & 1380 & 181 & $3,5 \mathrm{~min}$ & $\simeq 35 \mathrm{~kb}$ \\
7 & 3166 & 181 & $23 \mathrm{~min}$ & $\simeq 80 \mathrm{~kb}$ \\
8 & 6835 & 181 & 2,6 hours & $\simeq 200 \mathrm{~kb}$ \\
9 & 13993 & 271 & 27 hours & $\simeq 500 \mathrm{~kb}$ \\
10 & 27398 & 271 & 8 days & $\simeq 1 \mathrm{Mb}$ \\
11 & 51566 & $?$ & $?$ & $?$ \\
\hline
\end{tabular}

TABLE 2. Interpolation timings 
We performed our calculations with the computer algebra system MAGMA [4] for 62 out of the 63 invariants given in Table 1. The exception is the invariant of largest degree $\boldsymbol{j}_{10,1}$. Table 2 gives some indication of the accuracy required in our computations. The timings in it are for a single invariant of degree $d$, on a computer based on a single INTEL i7 $-2.80 \mathrm{GHz}$ processor. The sign "?" means that the computation did not finish within the time allotted to it.

As a result, we found for instance that

$$
\begin{aligned}
& 7^{2} \times \boldsymbol{j}_{0,2} \cdot \boldsymbol{I}_{9}^{2} / \boldsymbol{b}_{0}^{2}= 100 \boldsymbol{I}_{9}^{2}-300 \boldsymbol{I}_{18}, \\
& 7^{3} \times \boldsymbol{j}_{0,3} \cdot \boldsymbol{I}_{9}^{3} / \boldsymbol{b}_{0}^{3}=-1000 \boldsymbol{I}_{9}^{3}+4500 \boldsymbol{I}_{9} \boldsymbol{I}_{18}-13500 \boldsymbol{I}_{12} \boldsymbol{I}_{15}, \\
& 2^{5} \cdot 3^{4} \cdot 7^{2} \times \boldsymbol{j}_{2,0} \cdot \boldsymbol{I}_{9}^{2} / \boldsymbol{b}_{0}^{2}=2 \cdot 5 \cdot 7 \cdot 13 \boldsymbol{I}_{03}{ }^{4} \boldsymbol{I}_{06}-5 \boldsymbol{I}_{03}{ }^{3} \boldsymbol{I}_{09} \\
& \quad-3^{2} \cdot 5 \boldsymbol{I}_{03}{ }^{3} \boldsymbol{J}_{09}+2^{6} \cdot 5 \cdot 7 \cdot 29 \boldsymbol{I}_{03}{ }^{2} \boldsymbol{I}_{06}{ }^{2}-2 \cdot 5 \cdot 23 \boldsymbol{I}_{03}{ }^{2} \boldsymbol{I}_{12} \\
&+2^{2} \cdot 3 \cdot 5 \boldsymbol{I}_{03}{ }^{2} \boldsymbol{J}_{12}-2^{4} \cdot 3^{2} \cdot 5 \cdot 17 \boldsymbol{I}_{03} \boldsymbol{I}_{06} \boldsymbol{I}_{09}+2^{4} \cdot 3^{3} \cdot 5^{2} \boldsymbol{I}_{03} \boldsymbol{I}_{06} \boldsymbol{J}_{09} \\
&+2^{6} \cdot 5 \cdot 7 \boldsymbol{I}_{03} \boldsymbol{J}_{15}+2^{9} \cdot 3^{2} \cdot 5^{2} \cdot 7 \boldsymbol{I}_{06}{ }^{3}+2^{5} \cdot 3^{2} \cdot 5 \cdot 113 \boldsymbol{I}_{06} \boldsymbol{I}_{12} \\
& \quad-2^{6} \cdot 3^{2} \cdot 5^{2} \boldsymbol{I}_{06} \boldsymbol{J}_{12}+2^{5} \cdot 3^{2} \boldsymbol{I}_{09^{2}}{ }^{2}-2^{3} \cdot 3^{2} \cdot 5 \boldsymbol{I}_{18}-2^{3} \cdot 3^{2} \cdot 5 \cdot 7 \boldsymbol{J}_{18} .
\end{aligned}
$$

We observe that only very few primes divide the denominators of the coefficients, namely 2, 3 and 7 . In fact, for the 62 relations that we computed, only 11 primes occur in the denominators of the coefficients; these are 2, 3, 5, 7, 11, 13, 17, 23, 37, 79 and 89.

Remark 2.20. It is perhaps worth noting here that we do not need the full theoretical knowledge from Section 2.6 to obtain an inroad into our problem. Indeed, Proposition 2.10 gives us a bound on the denominator of $\boldsymbol{j}\left(\ell^{*}(\widetilde{F})\right) /\left(\boldsymbol{b}_{0}\left(\ell^{*}(\widetilde{F})\right)^{d}\right.$ since by Lemma 2.13 - (ii) this denominator divides $\boldsymbol{b}_{0}\left(\ell^{*}(\widetilde{F})\right)^{d}$. In principle, this bound on the degree of the denominator gives us all that we need to make the above linear algebra work, at the cost of a longer running time since our lack of theoretical knowledge makes us miss the presence of certain cancellations between the numerator and denominator.

Had we therefore lacked our particular knowledge of the denominator, we could, after finding some conjectural expressions in low degree, have proceeded under the assumption that the invariants were is of the form $\boldsymbol{P} / \boldsymbol{I}_{9}^{d}$, derived conjectural expressions for the $\boldsymbol{P}$, and then verified that these are indeed the only rational expressions that satisfy the given denominator bound and coincide with $\boldsymbol{j} \ell^{*} T /\left(\boldsymbol{b} \ell^{*} T\right)^{d}$ on a sufficiently large set of points. As this verification is relatively quick, this is a feasible approach. We include this remark because such an approach could still be useful in more general situations where fewer theoretical tools are available.

Remark 2.21. We did similar interpolation experiments for the locus of quartics on which either the covariant $\boldsymbol{T}$ or the covariant $\boldsymbol{X}$ of order 2 defined by (1.24) are normalized. This time, we have to use the equivariant map $\ell$ instead of $\ell^{*}$ because we are using covariants instead of contravariants ( $c f$. Section 2.1).

But a major issue is then that $\boldsymbol{T}$ and $\boldsymbol{X}$ are now of degree 5 rather than 4 . This causes the role of the invariant $\boldsymbol{I}_{9}$ in (2.58) to be taken over by invariants $\boldsymbol{I}_{T}$ and $\boldsymbol{I}_{X}$ of degree 21 . While we have been able to determine the corresponding interpolation polynomials $\boldsymbol{P}$ for the joint invariants of degree $\leq 4$, it now seems far more difficult to compute them for larger degrees. For $d=10$, the largest degree 
in which we could successfully compute when using the contravariant $\boldsymbol{\rho}$, this leads to a linear system of dimension 16893297 over $\mathbb{Q}$.

\section{RECONSTRUCTION}

In this section we will explain how to reconstruct a quartic curve from its Dixmier-Ohno invariants. After some theoretical considerations in Section 3.1, we give a first version of our reconstruction algorithms in Section 3.2. We briefly consider rationality issues that appear when working over a non-algebraically closed base field in Section 3.3. The concluding Section 3.4 gives an example and discusses the efficiency of our current implementation of these algorithms.

3.1. Stability. In Section 1.2, we have explained how to obtain the Dixmier-Ohno invariants of ternary quartic forms. Geometrically, this gives rise to a composed arrow

$$
\operatorname{Spec} K\left[\operatorname{Sym}^{4}\left(V^{*}\right)\right] \rightarrow \operatorname{Spec} K\left[\operatorname{Sym}^{4}\left(V^{*}\right)\right]^{\operatorname{SL}(V)} \hookrightarrow \mathbb{A}_{K}^{13} .
$$

On $K$-points, this sends a ternary quartic form $F$ over $K$ to its tuple of DixmierOhno invariants.

We will denote the closures of the image of (3.1) by $\mathbb{A}_{D O}$. Now it is not always true that the inverse image of an element of $\mathbb{A}_{\mathrm{DO}}(K)$ consists of a single $\mathrm{SL}(V)$ orbit of ternary quartic forms. If $F$ is a ternary quartic, we will say that it is stable if it belongs to the stable locus under the action of $\mathrm{SL}(V)$ in the sense of geometric invariant theory, i.e., if its orbit is uniquely determined by its invariants. This locus was characterized by Mumford (see [25, § 4]); its geometric points correspond to those quartics $F$ for which the curve $\mathrm{X}: F=0$ is reduced and has ordinary double points or cusps. This includes the locus of ternary quartics $F$ that define smooth plane quartic curves $X$.

As was sketched in the introduction, our way to reconstruct a ternary quartic form from its Dixmier-Ohno invariants is essentially to make a counterclockwise tour through the diagram

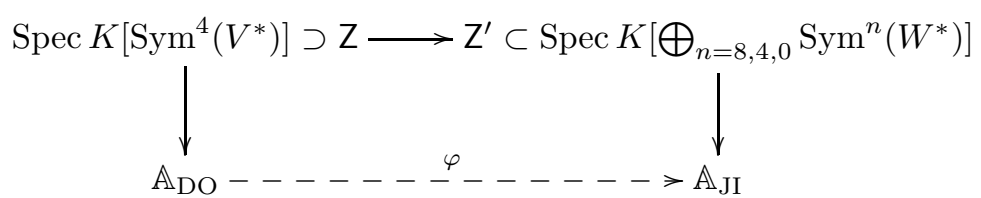

Here the space $\mathbb{A}_{J I}$ is the affine space corresponding to the generators of the ring of joint invariants in Section 1.3 along with the invariant $\boldsymbol{b}_{0}$; it is constructed as $\mathbb{A}_{D O}$ above. The top arrow is induced by the equivariant map $\ell^{*}$ from (2.10), the vertical arrows are canonical projection maps, and the bottom arrow $\varphi$ is the rational map described by the functions in Theorem 2.16.

As we want to recover a quartic $F$ from its invariants, we assume that $F$ is stable. Our method is also based on the assumption that $\boldsymbol{I}_{12}(F) \neq 0$. We can therefore assume that $F \in \mathrm{Z}(K)$ and consider the associated triple of binary forms $\underline{b}=\left(b_{8}, b_{4}, b_{0}\right) \in \mathrm{Z}^{\prime}(K) \subset \mathrm{Y}^{\prime}(K)$.

Since we have $\boldsymbol{u}(F) \neq 0$ for such an $F$, the map $\varphi$ is well-defined by Proposition 2.7. We denote the joint invariants that are the coordinates of the image of the Dixmier-Ohno invariants of $F$ by $j_{d_{1}, d_{2}}$. To analyze when our reconstruction can work, it is then enough to check when the $j_{d_{1}, d_{2}}$ determine a unique orbit of 
$\underline{b}$ under $\mathrm{SL}(W)$. Under the assumptions above, the following theorem proves that this is always the case.

Theorem 3.1. Let $F$ be a stable ternary quartic form such that $\boldsymbol{I}_{12}(F) \neq 0$ and let $j_{d_{1}, d_{2}}$ the joint invariants image of the Dixmier-Ohno invariants of $F$ by $\varphi$. Then the $j_{d_{1}, d_{2}}$ determine a unique triple $\underline{b}=\left(b_{8}, b_{4}, b_{0}\right)$ up to the action of $\mathrm{SL}(W)$.

As $b_{0}$ is a joint invariant, the proof comes down to determining when the orbits of the pair $\left(b_{8}, b_{4}\right)$ are uniquely determined by their joint invariants $\boldsymbol{j}_{d_{1}, d_{2}}$. This is the case if and only if $\underline{b}$ is in the stable locus of the action of $\mathrm{SL}(W)$ on $\operatorname{Sym}_{8}\left(W^{*}\right) \oplus$ $\operatorname{Sym}^{4}\left(W^{*}\right)$ in the sense of geometric invariant theory. We have the following result.

Lemma 3.2. An element $\left(b_{8}, b_{4}\right) \in \operatorname{Sym}^{8}\left(W^{*}\right) \oplus \operatorname{Sym}^{4}\left(W^{*}\right)$ is not stable for the action of $\mathrm{SL}(W)$ if and only if the forms $b_{8}$ and $b_{4}$ have a common root that is of multiplicity greater than 3 for $b_{8}$ and of multiplicity greater than 1 for $b_{4}$.

Proof. This can be proved by using the numerical stability criterion due to Hilbert and Mumford. We follow the setting of [10, Sec.9.4]. Considering the action of the maximal torus $\left[\begin{array}{cc}t & 0 \\ 0 & t^{-1}\end{array}\right]$ on $\left(b_{8}, b_{4}\right)$ shows that the set of weights of $\left(b_{8}, b_{4}\right)$ is contained in $S=\{-8,-6, \ldots, 6,8\}$. More precisely it is equal to

$$
w t\left(b_{8}, b_{4}\right)=\left\{2 i: i \text { such that } b_{8,4+i} \neq 0 \text { or } b_{4,2+i} \neq 0\right\},
$$

where we have written $b_{8}=\sum b_{8, i} z_{1}^{i} z_{2}^{8-i}$ and $b_{4}=\sum b_{4, i} z_{1}^{i} z_{2}^{4-i}$. By [10, Theorem 9.2], one knows that $\left(b_{8}, b_{4}\right)$ is stable for the action of this torus if and only if 0 belongs to the interior of the convex hull of $w t\left(b_{8}, b_{4}\right)$ in $\mathbb{R}$. Hence $\left(b_{8}, b_{4}\right)$ is not stable for this action if and only if

$$
\left\{\begin{array} { l } 
{ b _ { 8 , 8 } = b _ { 8 , 7 } = b _ { 8 , 6 } = b _ { 8 , 5 } = 0 , } \\
{ b _ { 4 , 4 } = b _ { 4 , 3 } = 0 , }
\end{array} \quad \text { or } \quad \left\{\begin{array}{l}
b_{8,0}=b_{8,1}=b_{8,2}=b_{8,3}=0, \\
b_{4,0}=b_{4,1}=0 .
\end{array}\right.\right.
$$

We denote the forms that satisfy one of these conditions by $U$. Now by $[10$, Theorem 9.3], we know that $\left(b_{8}, b_{4}\right)$ is not stable if and only if there exists a $T \in$ $\mathrm{SL}(W)$ such that $\left(b_{8}, b_{4}\right) . T \in U$. If $\left(b_{8}, b_{4}\right)$ is such that $b_{8}$ and $b_{4}$ have a common root that is of multiplicity greater than 3 for $b_{8}$ and greater than 1 for $b_{4}$, we can find an element $T$ of $\mathrm{SL}(W)$ that sends this root to $(0: 1)$, so that $\left(b_{8}, b_{4}\right) \cdot T \in$ $U$. Conversely, if $\left(b_{8}, b_{4}\right)$ is not stable, then there exists $T \in \mathrm{SL}(W)$ such that $\left(b_{8}, b_{4}\right) . T \in U$. This indeed implies that $b_{8}$ and $b_{4}$ have a common root with the specified multiplicities.

Proof of Theorem 3.1. Suppose that $\left(b_{8}, b_{4}\right)$ is not stable. Then up to the action of $\mathrm{SL}(W)$, we have $b_{8}=z_{1}^{4} \cdot c_{4}\left(z_{1}, z_{2}\right)$ with $c_{4}$ a universal form of degree 4 and $b_{4}=z_{1}^{2} \cdot c_{2}\left(z_{1}, z_{2}\right)$ with $c_{2}$ a universal form of degree 2 . The form $b_{0}$ can be chosen freely. We now consider the curve $F=\left(\ell^{*}\right)^{-1}(\underline{b})$, which depends on 9 parameters. A computation with MAGMA then shows that $F$ has a non-ordinary singularity at $(0: 0: 1)$.

3.2. Algorithms. Let $\underline{I}=\left(I_{3}, \ldots, I_{27}\right) \in \mathbb{A}_{\mathrm{DO}}(K)$ be a tuple of Dixmier-Ohno invariants, that is, an element of the image of the embedding (3.1). The methods of the previous section allow us to derive an algorithm to generically construct a ternary quartic form $F$ whose tuple of Dixmier-Ohno invariants equals $\underline{I}$.

We want to use Theorem 2.16. To make that possible, we make our first assumption. 
(i) We have $I_{12} \neq 0$.

Because of this, we may suppose that $F$ is an element of $Z$ and we can then use (2.43) with $T=$ Id to find a value of the constant $u \in K^{*}$. In light of (2.46) we can then calculate the exact value of any joint invariants $\boldsymbol{j} \ell^{*}(F)$ by evaluating the polynomials $\boldsymbol{P}$ computed by interpolation in Section 2.8 at the tuple $\underline{I}$. In the current implementation, we favor a different strategy in order to avoid the computation of the cube root $u$. Since we know how the Dixmier-Ohno invariants behave under scalar multiplication of the underlying forms, we can further assume without loss of generality that $F$ satisfies $\boldsymbol{b}_{0} \ell^{*}(F)=I_{9}$. Beyond avoiding a cube root, this simplifies the formula (2.47). If we are really interested in getting a form $F$ with Dixmier-Ohno invariants equal to $\underline{I}$ we will perform an extra step at the end of our algorithm, as we will mention later.

In this setting we get $\boldsymbol{j}_{2,0} \ell^{*}(F), \boldsymbol{j}_{3,0} \ell^{*}(F), \ldots \boldsymbol{j}_{10,0} \ell^{*}(F)$ as polynomial expressions in the $\underline{I}$. We now need to construct $b_{8}$ and $b_{4}$ in the image

$$
\ell^{*}(F)=\left(b_{8}, b_{4}, b_{0}\right) \in \operatorname{Sym}^{8}\left(W^{*}\right) \oplus \operatorname{Sym}^{4}\left(W^{*}\right) \oplus \operatorname{Sym}^{0}\left(W^{*}\right)
$$

with such joint invariants; note that $b_{0}$ is already given. We will rely on the following generic strategy. By Remark 1.8, we can compute the Shioda invariants of the binary octic $b_{8}$ and we can then reconstruct $b_{8}$ by applying the results of [22], at least as long as Shioda invariants separate $\mathrm{SL}(W)$-orbits. We thus add this genericity assumption.

(ii) The roots of $b_{8}$ have multiplicities less than 4 .

This assumption depends only on the invariants of $F$. More precisely, a calculation shows that we have to be outside the locus defined by

$$
\begin{array}{r}
49 \boldsymbol{j}_{3,0}^{2}-81 \boldsymbol{j}_{2,0}^{3}=33 \boldsymbol{j}_{4,0}-25 \boldsymbol{j}_{2,0}^{2}=27 \boldsymbol{j}_{5,0}-20 \boldsymbol{j}_{3,0} \boldsymbol{j}_{2,0}=0 \\
77 \boldsymbol{j}_{6,0}-50 \boldsymbol{j}_{2,0}^{3}=363 \boldsymbol{j}_{7,0}-125 \boldsymbol{j}_{3,0} \boldsymbol{j}_{2,0}^{2}=0 \\
\boldsymbol{j}_{8,0}=\boldsymbol{j}_{9,0}=\boldsymbol{j}_{10,0}=0
\end{array}
$$

Remark 3.3. We do not dig deeper into this matter here, but we plan to address the implementation of the full reconstruction problem in due time, especially to deal with the last assumption. For now one can treat the non-generic cases by using Gröbner basis calculations that make use of joint invariants with $b_{4}$.

Now the results from [22] return an octic $b_{8}^{\prime}$ with the given Shioda invariants up to scalar multiplication in a weighted projective space, as there is an action of $\mathrm{SL}(W)$ to play with. So there exists a scalar $\lambda$ such that the invariants of $\lambda b_{8}^{\prime}$ exactly equal the values $\boldsymbol{j}_{d, 0} \ell^{*}(F)$. Such a constant $\lambda$ can be obtained from the evaluations at $b_{8}^{\prime}$ of the invariants $\boldsymbol{j}_{d, 0}$.

Given $b_{0}=I_{9}$ and $b_{8}=\lambda b_{8}^{\prime}$, it remains to determine $b_{4}$. This determination simplifies under an additional generic assumption on the 14 joint invariants of degree 1 in the coefficients of $\boldsymbol{b}_{4}$

$$
\boldsymbol{j}_{2,1}, \boldsymbol{j}_{3,1}, \boldsymbol{j}_{4,1}, \boldsymbol{j}_{4,1}^{\prime}, \boldsymbol{j}_{5,1}, \boldsymbol{j}_{5,1}^{\prime}, \boldsymbol{j}_{6,1}, \boldsymbol{j}_{6,1}^{\prime}, \boldsymbol{j}_{7,1}, \boldsymbol{j}_{7,1}^{\prime}, \boldsymbol{j}_{8,1}, \boldsymbol{j}_{8,1}^{\prime}, \boldsymbol{j}_{9,1} \text { and } \boldsymbol{j}_{10,1}
$$

seen as linear forms in the coefficients of $\boldsymbol{b}_{4}$, namely:

(iii) The specialization of the linear forms (3.7) at $F$ generate a vector space of rank 5.

We can then find the coefficients of $b_{4}$ by solving a linear system. 
Remark 3.4. Experimentally, in the very rare cases where this assumption is not met, we complement these linear constraints with 5 equations of degree 4 that come from the relation

$$
\boldsymbol{\rho}\left(\left(\ell^{*}\right)^{-1}\left(\left(b_{8}, b_{4}, b_{0}\right)\right)\right)=u \cdot\left(v_{2}^{2}-v_{1} v_{3}\right) .
$$

Note that in addition to these $14+5=19$ equations, we may make use of the joint invariants of degree 2, 3 and 4 in $\boldsymbol{b}_{4}$ too. We have many equations of small degree in few unknowns. In such a situation, Gröbner basis calculations often yields the result.

Now that we have all the factors of the decomposition (3.5), we can determine a ternary quartic form $\widetilde{F}=\left(\ell^{*}\right)^{-1}\left(\left(b_{8}, b_{4}, b_{0}\right)\right)$. By construction, this quartic has the requested Dixmier-Ohno invariants seen as a point in the weighted projective space $\mathbb{P}(1: 2: 3: 3: 4: 4: 5: 5: 6: 6: 7: 7: 9)$.

This suffices for our geometric purposes, but let us still illustrate how to find a quartic form $F$ with Dixmier-Ohno invariants exactly equal $\underline{I}$, in the simplest case where $I_{3} \neq 0$. Compute $\lambda=I_{3} / \boldsymbol{I}_{3}(\widetilde{F})$ and let

$$
M=\left(\begin{array}{lll}
\lambda & 0 & 0 \\
0 & 1 & 0 \\
0 & 0 & 1
\end{array}\right) .
$$

Since the weight of a degree $3 d$ Dixmier-Ohno invariant is $4 d$ we see that for $F=\frac{1}{\lambda} \cdot \widetilde{F} \cdot M=\frac{1}{\lambda} \cdot \widetilde{F}\left(\lambda x_{1}, x_{2}, x_{3}\right)$ we get

$$
\boldsymbol{I}_{3}(F)=\operatorname{det}(M)^{4} \cdot \frac{\boldsymbol{I}_{3}(\widetilde{F})}{\lambda^{3}}=\lambda \cdot \boldsymbol{I}_{3}(\widetilde{F})=I_{3} .
$$

3.3. Descent. While the algorithm in the last section enables us to reconstruct generic ternary quartic forms with given Dixmier-Ohno invariants, it has some drawbacks that appear as soon as we try to work over a non-algebraically closed base field $k$ of characteristic 0 .

Under the genericity assumptions in the previous paragraph, the first additional condition that we meet for reconstruction to be possible over the base field is the existence of a binary octic $b_{8}^{\prime}$ as constructed in that paragraph with coefficients in $k$. That is, we have to assume that

$\left(^{*}\right)$ There exists a binary octic form $b_{8}^{\prime}$ over $k$ whose tuple of Shioda invariants is equivalent to the Shioda invariants calculated from the given Dixmier-Ohno invariants.

If there exists such a $b_{8}^{\prime}$, then our main worry is to ensure that the scalar $\lambda$ is $k$-rational. Since all invariants involved are rational, an argument as in $[22, \S 1.4]$ is true under the following additional generic assumption:

(gen) The weights of the entries at which the Shioda invariants are nonzero forms a set whose elements generate the unit ideal in $\mathbb{Z}$.

The condition (gen) is generically satisfied. On the other hand, the condition $\left(^{*}\right)$ is more subtle. Over number fields, it is in general impossible to avoid a quadratic extension when applying the above approach directly (on the other hand, such an obstruction never occurs over finite fields). Indeed, our reconstruction always returns a ternary quartic $F$ such that the conic associated to $\boldsymbol{\rho}(F)$ admits a rational 
point over its field of definition. Hence, if we start from a quartic $F$ over $k$ without geometric automorphism and such that $\boldsymbol{\rho}(F)$ has no $k$-rational point, such a quadratic extension will necessarily occur.

So even when starting with a tuple of Dixmier-Ohno invariants that corresponds to a quartic curve over $k$, the algorithms above may not return a model over $k$ straight away; we usually need to pass to a quadratic extension. However, by tinkering with Weil cocycles, we can often still obtain a model over $k$, as is sketched in the following (artificial) example. We only scratch the surface of the required theory; more information can be found in [23] and in the seminal work [43].

Example 3.5. Let $\alpha=\sqrt{2}$ and consider the quartic curve $X$ defined by the polynomial $f$ given by

$$
\begin{aligned}
& 2 x_{1}^{4}+(4 \alpha+4) x_{1}^{3} x_{2}+x_{1}^{3} x_{3}+18 x_{1}^{2} x_{2}^{2}+(2 \alpha+1) x_{1}^{2} x_{2} x_{3}+x_{1}^{2} x_{3}^{2}+(8 \alpha+4) x_{1} x_{2}^{3} \\
+ & (2 \alpha+2) x_{1} x_{2}^{2} x_{3}+(\alpha+1) x_{1} x_{2} x_{3}^{2}+x_{1} x_{3}^{3}+5 x_{2}^{4}+2 x_{2}^{3} x_{3}+\alpha x_{2}^{2} x_{3}^{2}+\alpha x_{2} x_{3}^{3}-x_{3}^{4} .
\end{aligned}
$$

Then $X$ is isomorphic to its Galois conjugate $X^{\sigma}$ via the matrix

$$
T=\left(\begin{array}{ccc}
1 & 2 \alpha+4 & 0 \\
0 & -2 \alpha-3 & 0 \\
0 & 0 & 1
\end{array}\right)
$$

so that $f^{\sigma}=f . T$. Explicitly, the matrix $T$ can be found by similar methods as those developed in this paper, namely by normalizing both $f$ and $f^{\sigma}$ and thus reducing the problem to a question of $\mathrm{SL}(W)$-equivalence.

The matrix $T$ satisfies the cocycle relation $T T^{\sigma}=1$ in $\operatorname{GL}(V)$. This is not automatically the case, and one needs to consider the induced morphisms between spaces of differentials in general; $c f$. the analogous case for twists in [13]. By Hilbert's Theorem 90 we can find a matrix $T_{0}$ over $\mathbb{Q}(\alpha)$ such that $T=T_{0}^{-1} T_{0}^{\sigma}$, either by using a probabilistic method analogous to that employed in $[23, \S 4.1]$ or writing out the entries of $T$ with respect to a $\mathbb{Q}$-basis of $\mathbb{Q}(\alpha)$ and solving the corresponding linear system. Regardless, a solution is given by

$$
T_{0}=\left(\begin{array}{ccc}
1 & \alpha & 0 \\
1 & 1 & 0 \\
0 & 0 & 1
\end{array}\right) .
$$

We then have $\left(f \cdot T_{0}^{-1}\right)^{\sigma}=f^{\sigma} \cdot T_{0}^{-\sigma}=f^{\sigma} \cdot T^{-1} \cdot T_{0}^{-1}=f \cdot T_{0}^{-1}$, so that $f \cdot T_{0}^{-1}$ is Galois invariant. And indeed

$$
f . T_{0}^{-1}=x_{1}^{4}+x_{1}^{2} x_{2} x_{3}+x_{1} x_{2} x_{3}^{2}+x_{1} x_{3}^{3}+x_{2}^{4}-x_{3}^{4} .
$$

Remark 3.6. In fact any smooth plane quartic with trivial geometric automorphism group descends to its field of moduli, as follows immediately from the criterion in [43]. So starting with a $k$-rational point of invariants $\underline{I} \in \mathbb{P}_{\mathrm{DO}}(k)$ of the projective space $\mathbb{P}_{\mathrm{DO}}$ corresponding to $\mathbb{A}_{\mathrm{DO}}(k)$, we can generically obtain a reconstruction over $k$ by first finding one over a quadratic extension by using our algorithms at the beginning of this section and then following the same method as in Example 3.5.

So if the geometric automorphism group of the curve corresponding to $\underline{I}$ is trivial, then we can reconstruct over the field of moduli $k$. When the characteristic of $k$ is either 0 or large enough, then the results of [23] show that we can also obtain a plane quartic over $k$ corresponding to $\underline{I}$ if $\underline{I}$ corresponds to a plane quartic whose automorphism group has order strictly larger than 2 . 
It remains to discuss the case of smooth plane quartics with automorphism group $\mathbb{Z} / 2 \mathbb{Z}$. These admit a model over $K$ of the form

$$
X: x_{1}^{4}+f\left(x_{2}, x_{3}\right) x_{1}^{2}+g\left(x_{2}, x_{3}\right)=0 .
$$

It can be shown that if such a curve comes from a tuple $\underline{I} \in P_{\mathrm{DO}}(k)$, then the isomorphisms of $X$ with its conjugates induce a well-defined cocycle of automorphisms on the fixed line $x_{1}=0$, which is isomorphic to $\mathbb{P}^{1}$. This cocycle gives rise to a model $C$ of $\mathbb{P}^{1}$ over $k$, which is $k$-isomorphic to a conic over $k$. It is then possible to obtain a plane quartic over $k$ with invariants $\underline{I}$ if and only if the conic $C$ has a $k$-rational point. We hope to treat this subject more fully in future work; it is a generalization of the descent from $\mathbb{C}$ to its subfield $\mathbb{R}$ considered in [1] and allows for a full classification of curves that do not descend to their field of moduli.

3.4. Implementation. We conclude this article by briefly discussing the implementation and efficiency of our implementation. To do this, we give a single example of our methods. We follow the procedure described in Section 3.3 for the Dixmier-Ohno invariants

$$
\begin{aligned}
\underline{I}=\left(0,0,0,0,-\frac{7 \cdot 19}{2^{14} \cdot 3^{8} \cdot 5^{2}}, 0,\right. & -\frac{11 \cdot 19}{2^{17} \cdot 3^{10} \cdot 5^{2}}, 0, \frac{7 \cdot 19^{2}}{2^{20} \cdot 3^{11} \cdot 5^{3}}, \frac{19^{2}}{2^{20} \cdot 3^{11} \cdot 5^{3}}, \\
& \left.-\frac{19^{2} \cdot 31}{2^{24} \cdot 3^{13} \cdot 5^{5}},-\frac{17 \cdot 19^{2}}{2^{21} \cdot 3^{12} \cdot 5^{5}},-\frac{19^{2} \cdot 6553}{2^{39} \cdot 3^{6} \cdot 5^{5} \cdot 11}\right) .
\end{aligned}
$$

We obtained this tuple by looking for a small rational point $\left(I_{3}, I_{6}, \ldots, I_{27}\right)$ in the space $\mathbb{A}^{\mathrm{DO}}(\mathbb{Q}) \cap \operatorname{im} \varphi$ with $I_{12} \neq 0, I_{27} \neq 0$. We therefore did not a priori know a curve with these invariants.

While these invariants are simple enough to write down, the intermediate expressions calculated over $\mathbb{Q}$ in the algorithm have very large coefficients. We therefore switch to the finite field $\mathbb{F}_{29}$ and perform our computation there first. We have $\underline{I} \bmod 29=(0,0,0,0,17,0,17,0,2,21,4,4,9)$. Applying Theorem 2.16 by using our interpolation polynomial gives us the joint invariants

$$
S(\underline{I})=\left(j_{2,0}, j_{3,0}, \ldots, j_{10,0}\right)=(4,19,4,20,19,9,24,24,8) .
$$

A corresponding binary octic $b_{8}$ is

$$
15 y_{1}^{8}+9 y_{1}^{7} y_{2}+6 y_{1}^{6} y_{2}^{2}+19 y_{1}^{5} y_{2}^{3}+28 y_{1}^{4} y_{2}^{4}+16 y_{1}^{3} y_{2}^{5}+4 y_{1}^{2} y_{2}^{6}+25 y_{1} y_{2}^{7}+20 y_{2}^{8}
$$

for which

$$
S\left(b_{8}\right)=(7,9,5,12,18,8,23,18,11) .
$$

We have $S\left(b_{8}\right)=\left(\lambda^{2} j_{2,0}, \ldots, \lambda^{10} j_{10,0}\right)$ with $\lambda=26$.

We have, $b_{0}=I_{9}=0$ and we replace $b_{8}$ by $\lambda b_{8}=26 b_{8}$. It remains to determine the coefficients of $b_{4}=a_{4,0} y_{1}^{4}+a_{3,1} y_{1}^{3} y_{2}+a_{2,2} y_{1}^{2} y_{2}^{2}+a_{1,3} y_{1} y_{2}^{3}+a_{0,4} y_{2}^{4}$. Using the joint invariants, scaled by $\lambda$, leads to the system of equations

$$
\begin{aligned}
13 a_{4,0}+21 a_{3,1}+4 a_{2,2}+17 a_{1,3}+25 a_{0,4}+9 & =0, \\
9 a_{4,0}+8 a_{3,1}+10 a_{2,2}+19 a_{1,3}+25 a_{0,4}+18 & =0, \\
9 a_{4,0}+22 a_{3,1}+7 a_{2,2}+18 a_{1,3}+22 a_{0,4}+17 & =0, \\
22 a_{4,0}+15 a_{3,1}+3 a_{2,2}+a_{1,3}+19 a_{0,4}+18 & =0, \\
23 a_{4,0}+10 a_{3,1}+19 a_{2,2}+28 a_{1,3}+22 a_{0,4}+26 & =0, \\
13 a_{4,0}+25 a_{3,1}+8 a_{2,2}+12 a_{1,3}+3 a_{0,4}+16 & =0 .
\end{aligned}
$$

This system admits the unique solution

$$
b_{4}=9 y_{1}^{4}+8 y_{1}^{3} y_{2}+19 y_{1}^{2} y_{2}^{2}+17 y_{1} y_{2}^{3}+23 y_{2}^{4}
$$


and we get that the reconstruction $F=\left(\ell^{*}\right)^{-1}\left(\left(b_{8}, b_{4}, b_{0}\right)\right)$ defines a curve given by

$$
\begin{aligned}
24 x_{1}^{4}+ & 13 x_{1}^{3} x_{2}+x_{1}^{3} x_{3}+21 x_{1}^{2} x_{2}^{2}+22 x_{1}^{2} x_{2} x_{3}+28 x_{1}^{2} x_{3}^{2}+7 x_{1} x_{2}^{3}+23 x_{1} x_{2}^{2} x_{3} \\
& +27 x_{1} x_{2} x_{3}^{2}+10 x_{1} x_{3}^{3}+4 x_{2}^{4}+24 x_{2}^{3} x_{3}+2 x_{2}^{2} x_{3}^{2}+20 x_{2} x_{3}^{3}+3 x_{3}^{4}
\end{aligned}
$$

Over $\mathbb{Q}$, our algorithms returns on input (3.16) a quartic with coefficients that have around 100 decimal digits. This inefficiency seems to stem from the intermediate reconstruction from Shioda invariants. But by using reduction theory (see the algorithms in [37] and their implementation in MAGMA), we can obtain the much nicer quartic

$$
\begin{array}{r}
4 x_{1}^{4}-12 x_{1}^{3} x_{2}-62 x_{1}^{3} x_{3}-108 x_{1}^{2} x_{2}^{2}+144 x_{1}^{2} x_{2} x_{3}+12 x_{1}^{2} x_{3}^{2}+20 x_{1} x_{2}^{3} \\
-90 x_{1} x_{2}^{2} x_{3}-210 x_{1} x_{2} x_{3}^{2}+125 x_{1} x_{3}^{3}-30 x_{2}^{4}-160 x_{2}^{3} x_{3}+135 x_{2} x_{3}^{3}+180 x_{3}^{4}
\end{array}
$$

Over a finite field the above coefficient explosion that we mentioned above is not an issue, and our algorithms are correspondingly fast there. Their performances is recorded in Table 3 (average timings on an INTEL i7 - 2.80 GHz processor with Magma 2.21-10). Over finite fields, it is not known to us when the Dixmier-Ohno invariants are generators of the ring of invariants but we can at least verify a posteriori whether the constructed quartic has the correct Dixmier-Ohno invariants.

\begin{tabular}{l|l|l|l|l|l} 
& $\mathbb{F}_{p}$ & $\mathbb{F}_{p^{25}}$ & $\mathbb{F}_{p^{50}}$ & $\mathbb{F}_{p^{75}}$ & $\mathbb{F}_{p^{100}}$ \\
\hline \hline$p=41$ & $0.05 \mathrm{~s}$ & $0.5 \mathrm{~s}$ & $0.8 \mathrm{~s}$ & $1.2 \mathrm{~s}$ & $2.8 \mathrm{~s}$ \\
\hline & $\mathbb{F}_{p}$ & $\mathbb{F}_{p^{2}}$ & $\mathbb{F}_{p^{3}}$ & $\mathbb{F}_{p^{4}}$ & $\mathbb{F}_{p^{10}}$ \\
\hline \hline$p \approx 10^{50}$ & $0.2 \mathrm{~s}$ & $0.6 \mathrm{~s}$ & $0.9 \mathrm{~s}$ & $1.2 \mathrm{~s}$ & $3.1 \mathrm{~s}$ \\
$p \approx 10^{100}$ & $0.2 \mathrm{~s}$ & $0.8 \mathrm{~s}$ & $1.1 \mathrm{~s}$ & $1.6 \mathrm{~s}$ & $5.9 \mathrm{~s}$ \\
$p \approx 10^{150}$ & $0.3 \mathrm{~s}$ & $1.0 \mathrm{~s}$ & $1.6 \mathrm{~s}$ & $2.3 \mathrm{~s}$ & $9.8 \mathrm{~s}$ \\
$p \approx 10^{200}$ & $0.4 \mathrm{~s}$ & $1.5 \mathrm{~s}$ & $2.6 \mathrm{~s}$ & $3.7 \mathrm{~s}$ & $18.4 \mathrm{~s}$ \\
\hline
\end{tabular}

TABLE 3. Reconstruction timings

\section{REFERENCES}

[1] M. Artebani and S. Quispe. Fields of moduli and fields of definition of odd signature curves. Arch. Math., 99(4):333-344, 2012.

[2] R. Basson. Arithmétique des espaces de modules des courbes hyperelliptiques de genre 3 en caractéristique positive. PhD thesis, Université de Rennes 1, Rennes, 2015.

[3] C. Böhning. The rationality of the moduli space of curves of genus 3 after P. Katsylo. In Cohomological and geometric approaches to rationality problems, volume 282 of Progr. Math., pages 17-53. Birkhäuser Boston, Inc., Boston, MA, 2010.

[4] W. Bosma, J. Cannon, and C. Playoust. The Magma algebra system. I. The user language. J. Symbolic Comput., 24(3-4):235-265, 1997. Computational algebra and number theory (London, 1993).

[5] A. E. Brouwer and M. Popoviciu. The invariants of the binary decimic. J. Symbolic Comput., 45(8):837-843, 2010.

[6] A. E. Brouwer and M. Popoviciu. The invariants of the binary nonic. J. Symbolic Comput., 45(6):709-720, 2010.

[7] A. Clebsch. Theorie der binären algebraischen formen. Verlag von B.G. Teubner, Leipzig, 1872. 
[8] H. Cohen, G. Frey, R. Avanzi, C. Doche, T. Lange, K. Nguyen, and F. Vercauteren, editors. Handbook of elliptic and hyperelliptic curve cryptography. Discrete Mathematics and its Applications (Boca Raton). Chapman \& Hall/CRC, Boca Raton, FL, 2006.

[9] J. Dixmier. On the projective invariants of quartic plane curves. Adv. in Math., 64:279-304, 1987.

[10] I. Dolgachev. Lectures on invariant theory, volume 296 of London Mathematical Society Lecture Note Series. Cambridge University Press, Cambridge, 2003.

[11] A.-S. Elsenhans. Explicit computations of invariants of plane quartic curves. J. Symbolic Comput., 68(part 2):109-115, 2015.

[12] W. Fulton and J. Harris. Representation theory, volume 129 of Graduate Texts in Mathematics. Springer-Verlag, New York, 1991. A first course, Readings in Mathematics.

[13] E. L. García. Twists of non-hyperelliptic curves. Preprint.

[14] V. Gatti and E. Viniberghi. Spinors of 13-dimensional space. Adv. in Math., 30(2):137-155, 1978.

[15] M. Girard and D. R. Kohel. Classification of genus 3 curves in special strata of the moduli space. Hess, Florian (ed.) et al., Algorithmic number theory. 7th international symposium, ANTS-VII, Berlin, Germany, July 23-28, 2006. Proceedings. Berlin: Springer. Lecture Notes in Computer Science 4076, 346-360 (2006)., 2006.

[16] P. Gordan. Beweis, dass jede Covariante und Invariante einer binären Form eine ganze Function mit numerischen Coefficienten einer endlichen Anzahl solcher Formen ist. Journal für die reine und angewandte Mathematik, 69:323-354, 1868.

[17] M. Hashimoto. Equivariant total ring of fractions and factoriality of rings generated by semiinvariants. Comm. Algebra, 43(4):1524-1562, 2015.

[18] D. Hilbert. Theory of algebraic invariants. Cambridge University Press, Cambridge, 1993. Translated from the German and with a preface by Reinhard C. Laubenbacher, Edited and with an introduction by Bernd Sturmfels.

[19] P. Katsylo. Rationality of the moduli variety of curves of genus 3. Comment. Math. Helv., 71(4):507-524, 1996.

[20] P. I. Katsylo. On the birational geometry of the space of ternary quartics. In Lie groups, their discrete subgroups, and invariant theory, volume 8 of Adv. Soviet Math., pages 95-103. Amer. Math. Soc., Providence, RI, 1992.

[21] H. Kraft and C. Procesi. Classical invariant theory. A primer, 1996.

[22] R. Lercier and C. Ritzenthaler. Hyperelliptic curves and their invariants: geometric, arithmetic and algorithmic aspects. J. Algebra, 372:595-636, 2012.

[23] R. Lercier, C. Ritzenthaler, F. Rovetta, and J. Sijsling. Parametrizing the moduli space of curves and applications to smooth plane quartics over finite fields. LMS J. Comput. Math., 17(suppl. A):128-147, 2014.

[24] R. Lercier, C. Ritzenthaler, and J. Sijsling. quartic_reconstruction; a Magma package for reconstructing plane quartics. https://github.com/JRSijsling/quartic_reconstruction/, 2016.

[25] E. Looijenga. Invariants of quartic plane curves as automorphic forms. In Algebraic geometry, volume 422 of Contemp. Math., pages 107-120. Amer. Math. Soc., Providence, RI, 2007.

[26] T. Maeda. On the invariant field of binary octavics. Hiroshima Mathematical Journal, 20(3):619-632, 1990.

[27] J.-F. Mestre. Construction de courbes de genre 2 à partir de leurs modules. In Effective methods in algebraic geometry, volume 94 of Prog. Math., pages 313-334, Boston, 1991. Birkäuser.

[28] T. Ohno. The graded ring of invariants of ternary quartics I, 2005 ? unpublished.

[29] M. Olive. About Gordan's algorithm for binary forms, 2014.

[30] P. J. Olver. Classical invariant theory, volume 44 of London Mathematical Society Student Texts. Cambridge University Press, Cambridge, 1999.

[31] V. L. Popov. Stability of the action of an algebraic group on an algebraic variety. Izv. Akad. Nauk SSSR Ser. Mat., 36:371-385, 1972.

[32] C. Procesi. Invariant theory, volume 2 of Monografías del Instituto de Matemática y Ciencias Afines [Monographs of the Institute of Mathematics and Related Sciences]. Instituto de Matemática y Ciencias Afines, IMCA, Lima, 1998. 
[33] K. Rökaeus. Computer search for curves with many points among abelian covers of genus 2 curves. In Arithmetic, geometry, cryptography and coding theory, volume 574 of Contemp. Math., pages 145-150. Amer. Math. Soc., Providence, RI, 2012.

[34] G. Salmon. A treatise on the analytic geometry of three dimensions. Vol. II. Fifth edition. Edited by Reginald A. P. Rogers. Chelsea Publishing Co., New York, 1965.

[35] T. Shioda. On the graded ring of invariants of binary octavics. American J. of Math., 89(4):1022-1046, 1967.

[36] T. A. Springer. Invariant theory. Lecture Notes in Mathematics, Vol. 585. Springer-Verlag, Berlin-New York, 1977.

[37] M. Stoll. Reduction theory of point clusters in projective space. Groups Geom. Dyn., 5(2):553$565,2011$.

[38] B. Sturmfels. Algorithms in invariant theory. Texts and Monographs in Symbolic Computation. SpringerWienNewYork, Vienna, second edition, 2008.

[39] M. van Leeuwen, A. Cohen, and B. Lisser. LiE, A Package for Lie Group Computations. Computer Algebra Nederland, Amsterdam, 1997.

[40] S. M. van Rijnswou. Testing the equivalence of planar curves. PhD thesis, Technische Universiteit Eindhoven, Eindhoven, 2001.

[41] F. von Gall. Das vollständige Formensystem einer binären Form achter Ordnung. Matematische Annalen, 17:31-51, 139-152, 456, 1880.

[42] F. Von Gall. Das vollständige Formensystem der binären Form 7ter Ordnung. Math. Ann., 31:318-336, 1888.

[43] A. Weil. The field of definition of a variety. American Journal of Mathematics, 78:509-524, 1956.

DGA Mi, La Roche Marguerite, 35174 Bruz, France.

IRMaR, Université de Rennes 1, Campus de Beaulieu, 35042 Rennes, France.

E-mail address: reynald.lercier@m4x.org

IRmaR, Université de Rennes 1, Campus de Beaulieu, 35042 Rennes, France.

E-mail address: christophe.ritzenthaler@univ-rennes1.fr

Department of Mathematics, Dartmouth College, 6188 Kemeny Hall, Hanover, NH 03755 USA

Institut für Reine Mathematik, Universität Ulm, Helmholtzstrasse 18, 89081 Ulm, Germany

E-mail address: sijsling@gmail.com 